\title{
The clustering of galaxies in the completed SDSS-III Baryon Oscillation Spectroscopic Survey: towards a computationally efficient analysis without informative priors
}

\author{
Marcos Pellejero-Ibanez, ${ }^{1,2,3,4 \star}$ Chia-Hsun Chuang, ${ }^{3,4}$ J. A. Rubiño-Martín, ${ }^{1,2}$ \\ Antonio J. Cuesta, ${ }^{5}$ Yuting Wang, ${ }^{6,7}$ Gongbo Zhao, ${ }^{6,7}$ Ashley J. Ross,,${ }^{7,8}$ \\ Sergio Rodríguez-Torres, ${ }^{3,9,10}$ Francisco Prada, ${ }^{3,9,11}$ Anže Slosar, ${ }^{12}$ \\ Jose A. Vazquez, ${ }^{12}$ Shadab Alam, ${ }^{13,14}$ Florian Beutler, ${ }^{7,15}$ Daniel J. Eisenstein, ${ }^{16}$ \\ Héctor Gil-Marín, ${ }^{17,18}$ Jan Niklas Grieb, ${ }^{19,20}$ Shirley Ho, ${ }^{13,14}$ \\ Francisco-Shu Kitaura, ${ }^{4,15,21}$ Will J. Percival, ${ }^{7}$ Graziano Rossi, ${ }^{22}$ \\ Salvador Salazar-Albornoz, ${ }^{19,20}$ Lado Samushia,,${ }^{7,23,24}$ Ariel G. Sánchez, ${ }^{20}$ \\ Siddharth Satpathy, ${ }^{13,14}$ Hee-Jong Seo, ${ }^{25}$ Jeremy L. Tinker ${ }^{26}$ Rita Tojeiro, ${ }^{27}$ \\ Mariana Vargas-Magaña, ${ }^{28}$ Joel R. Brownstein, ${ }^{29}$ Robert C Nichol $^{7}$ \\ and Matthew D Olmstead ${ }^{30}$
}

Affiliations are listed at the end of the paper

\begin{abstract}
We develop a new computationally efficient methodology called double-probe analysis with the aim of minimizing informative priors (those coming from extra probes) in the estimation of cosmological parameters. Using our new methodology, we extract the dark energy model-independent cosmological constraints from the joint data sets of the Baryon Oscillation Spectroscopic Survey (BOSS) galaxy sample and Planck cosmic microwave background (CMB) measurements. We measure the mean values and covariance matrix of $\left\{R, l_{\mathrm{a}}, \Omega_{\mathrm{b}} h^{2}, n_{\mathrm{s}}, \log \left(A_{\mathrm{s}}\right), \Omega_{\mathrm{k}}, H(z), D_{\mathrm{A}}(z), f(z) \sigma_{8}(z)\right\}$, which give an efficient summary of the Planck data and two-point statistics from the BOSS galaxy sample. The CMB shift parameters are $R=\sqrt{\Omega_{\mathrm{m}} H_{0}^{2}} r\left(z_{*}\right)$ and $l_{\mathrm{a}}=\pi r\left(z_{*}\right) / r_{\mathrm{s}}\left(z_{*}\right)$, where $z_{*}$ is the redshift at the last scattering surface, and $r\left(z_{*}\right)$ and $r_{\mathrm{s}}\left(z_{*}\right)$ denote our comoving distance to the $z_{*}$ and sound horizon at $z_{*}$, respectively; $\Omega_{\mathrm{b}}$ is the baryon fraction at $z=0$. This approximate methodology guarantees that we will not need to put informative priors on the cosmological parameters that galaxy clustering is unable to constrain, i.e. $\Omega_{\mathrm{b}} h^{2}$ and $n_{\mathrm{s}}$. The main advantage is that the computational time required for extracting these parameters is decreased by a factor of 60 with respect to exact full-likelihood analyses. The results obtained show no tension with the flat $\Lambda$ cold dark matter $(\Lambda \mathrm{CDM})$ cosmological paradigm. By comparing with the full-likelihood exact analysis with fixed dark energy models, on one hand we demonstrate that the double-probe method provides robust cosmological parameter constraints that can be conveniently used to study dark energy models, and on the other hand we provide a reliable set of measurements assuming dark energy models to be used, for example, in distance estimations. We extend our study to measure the sum of the neutrino mass using different methodologies, including double-probe analysis (introduced in this study), full-likelihood analysis and single-probe analysis. From full-likelihood
\end{abstract}


analysis, we obtain $\Sigma m_{v}<0.12$ (68 per cent), assuming $\Lambda$ CDM and $\Sigma m_{v}<0.20$ (68 per cent) assuming owCDM. We also find that there is degeneracy between observational systematics and neutrino masses, which suggests that one should take great care when estimating these parameters in the case of not having control over the systematics of a given sample.

Key words: cosmological parameters-distance scale-large-scale structure of Universecosmology: observations.

\section{INTRODUCTION}

We have entered the era of precision cosmology thanks to a dramatic increase in sky surveys, such as those for the cosmic microwave background (CMB; e.g. Bennett et al. 2013; Ade et al. 2014a), supernovae (SNe; Riess et al. 1998; Perlmutter et al. 1999; Conley et al. 2011; Sako et al. 2014), weak lensing (e.g. see Van Waerbeke \& Mellier 2003 for a review, Heymans et al. 2012; Erben et al. 2013) and large-scale structure (LSS) from galaxy redshift surveys, e.g. 2dF Galaxy Redshift Survey (Colless et al. 2001, 2003), Sloan Digital Sky Survey (SDSS; York et al. 2000; Abazajian et al. 2009), WiggleZ (Drinkwater et al. 2010; Parkinson et al. 2012) and the Baryon Oscillation Spectroscopic Survey (BOSS; Dawson et al. 2013; Alam et al. 2015a) of the SDSS-III (Eisenstein et al. 2011). The future galaxy redshift surveys, e.g. Euclid ${ }^{1}$ (Laureijs et al. 2011), Dark Energy Spectroscopic Instrument ${ }^{2}$ (Schlegel et al. 2011) and WFIRST ${ }^{3}$ (Green et al. 2012), will collect an order of magnitude more data. It is then critical to develop methodologies able to reliably extract the cosmological information from such large amount of data.

For recent cosmological SDSS analyses such as those of DR10 and DR11, we refer to Anderson et al. (2014), Beutler et al. (2014a), Samushia et al. (2014), Chuang et al. (2016b), Sanchez et al. (2014), Reid et al. (2014) and Alam et al. (2015b) for a gravity model test, Ross et al. (2014) for colour dependences and Tojeiro et al. (2014) for a low-redshift study. For earlier analyses, one can find the references in the papers listed above.

Eisenstein et al. (2005) proved the feasibility of measuring $\Omega_{\mathrm{m}} h^{2}$ and an effective distance, $D_{\mathrm{V}}(z)$, from the SDSS DR3 (Abazajian et al. 2005) Luminous Red Galaxies (LRG's), where $D_{\mathrm{V}}(z)$ corresponds to a combination of Hubble expansion rate $H(z)$ and angular diameter distance $D_{\mathrm{A}}(z)$, which will be defined in Section 5. Moreover, Chuang \& Wang (2012) showed how to measure $H(z)$ and $D_{\mathrm{A}}(z)$ simultaneously using the galaxy clustering data from the twodimensional two-point correlation function of SDSS DR7 (Abazajian et al. 2009) LRGs. This has been upgraded in Chuang \& Wang (2013a,b) with improved methodology and modelling to measure $H(z), D_{\mathrm{A}}(z)$, the normalized growth rate $f(z) \sigma_{8}(z)$ and the physical matter density $\Omega_{\mathrm{m}} h^{2}$ from the same data. Extra analyses have been performed to measure $H(z), D_{\mathrm{A}}(z)$ and $f(z) \sigma_{8}(z)$ from previous data releases of the SDSS BOSS galaxy sample such as those of Reid et al. (2012), Chuang et al. (2013, 2016b), Wang (2014), Anderson et al. (2014), Beutler et al. (2014a) and Samushia et al. (2014). In this work, we use Chuang \& Wang (2013a,b) and (Chuang et al. 2013, 2016a, companion paper) as the main references to determine $H(z), D_{\mathrm{A}}(z)$ and $f(z) \sigma_{8}(z)$.

If one wants to combine the likelihoods coming from the CMB and LSS, there are several ways in which we can approach the problem. The first and most obvious is to directly multiply them taking all data and correlations between parameters to end up with a

\footnotetext{
${ }^{1} \mathrm{http}: / /$ sci.esa.int/euclid

${ }^{2}$ http://desi.lbl.gov/

${ }^{3}$ http://wfirst.gsfc.nasa.gov/
}

posterior distribution of the parameters coming from the cosmological model under study. We dub this methodology 'full run' analysis. The main disadvantage of this is that, owing to the non-linear nature of gravity evolution, LSS models take too much time to be efficiently implemented into a Markov chain Monte Carlo (MCMC) based on Bayesian statistics code. A second and more common manner in the literature of dealing with the combination of these two likelihoods is to approximate the LSS likelihood. The usual way of doing this is by means of the measurement of LSS information given by the derived parameters $H(z), D_{\mathrm{A}}(z)$ and $f(z) \sigma_{8}(z)$ that will later be used for constraining dark energy cosmological models in combination with the CMB. Our concern here arises when one needs to set certain parameters, such as $\Omega_{\mathrm{b}} h^{2}, n_{\mathrm{s}}$ or even $\Omega_{\mathrm{m}} h^{2}$, to fixed or nearly fixed values to be able to find the aforementioned derived parameters. In the case in which one uses CMB prior knowledge (informative priors) for those unconstrained parameters, one could ask, when combining these results with the $\mathrm{CMB}$, whether we are double counting the information already used or missing weak degeneracies between these (nearly or completely fixed) parameters and those measured. To fix this, one might add some systematic error budget (e.g. see Anderson et al. 2014) or use very wide priors, e.g. $5 \sigma$ or $10 \sigma$ flat priors from CMB (see Chuang \& Wang 2012; Chuang, Wang \& Hemantha 2012), to minimize the potential systematic bias from informative priors. The latter would define what is called a 'singleprobe' method (SPM) as the only information used is that coming from LSS with no CMB assumptions involved. However, this approach would obtain weaker constraints owing to the wide priors. A third option, studied for the first time in this paper, is to approximate the full product of both the CMB and LSS likelihoods to obtain an efficient set of derived parameters that summarize the cosmological information content of the data sets. Certainly, as both likelihoods are taken together, one cannot miss degeneracies between LSS and the $\mathrm{CMB}$, and there will be no concern regarding prior fixed parameters on LSS because all those will be tightly constrained by the CMB. We call this methodology 'double probe', as opposed to 'single probe', where one uses a similar methodology for testing the LSS likelihood alone. Since CMB data have been proven to efficiently be summarized with a few parameters (e.g. see Wang \& Mukherjee 2007), we use the joint data set from Planck and BOSS to extract the cosmological constraints without fixing dark energy models.

Note that we assume that there is no early-time dark energy or dark energy clustering in this study. In principle, our methodology extracts the cosmological constraints from the joint data set in the optimal way since we do not need to include the uncertainty introduced by the informative priors.

In addition to constraining dark energy model parameters, we extend our study to limiting neutrino masses. High-energy physics experiments provide the squared mass differences between neutrino species from oscillation experiments. The latest results are $\Delta m_{21}^{2}=$ $7.53 \pm 0.18 \times 10^{-5} \mathrm{eV}^{2}$ and $\Delta m_{32}^{2}=2.44 \pm 0.06 \times 10^{-3} \mathrm{eV}^{2}$ for the normal hierarchy $\left(m_{3} \gg m_{2} \simeq m_{1}\right)$, and $\Delta m_{32}^{2}=2.52 \pm 0.07 \times$ $10^{-3} \mathrm{eV}^{2}$ for the inverted mass hierarchy $\left(m_{3} \ll m_{2} \simeq m_{1}\right.$; Olive \& 
Group 2014), where $m_{1,2,3}$ are the masses of the three neutrino species. Cosmology is a unique tool for the measurement of the sum of neutrino masses, $\Sigma m_{v}$, since this quantity affects the expansion rate and the way structures form and evolve. $\Sigma m_{v}$ estimates from galaxy clustering have been widely studied theoretically (see Hu, Eisenstein \& Tegmark 1998; Lesgourgues \& Pastor 2006 for a review) with different samples, such as WiggleZ (see RiemerSørensen, Parkinson \& Davis 2014; Cuesta, Niro \& Verde 2016a) or SDSS data (see Reid et al. 2010; Thomas, Abdalla \& Lahav 2010; Zhao et al. 2013; Beutler et al. 2014b; Aubourg et al. 2015). At late times, massive neutrinos can damp the formation of cosmic structure on small scales owing to the free-streaming effect (Dolgov 2002). Existing in the form of radiation in the early Universe, neutrinos shift the epoch of the matter-radiation equality, thus changing the shape of the CMB angular power spectrum. They affect the CMB via the so-called early integrated Sachs-Wolfe effect, which influences gravitational lensing measurements (e.g. Lesgourgues et al. 2006). Recent publications have attempted to constrain $\Sigma m_{v}$ by imposing upper limits (Seljak, Slosar \& McDonald 2006; Gong et al. 2008; Dunkley et al. 2009; Hinshaw et al. 2009; Ichiki, Takada \& Takahashi 2009; Li et al. 2009; Tereno et al. 2009; Reid et al. 2010; Komatsu et al. 2011; Saito, Takada \& Taruya 2011; de Putter et al. 2012; Sanchez et al. 2012; Xia et al. 2012; Giusarma et al. 2013) and some hints of lower limits using cluster abundance results (Burenin 2013; Rozo et al. 2013; Ade et al. 2014b; Battye \& Moss 2014; Wyman et al. 2014). We measure the sum of neutrino mass using different methodologies, including double-probe analysis (introduced in this study), full-likelihood analysis and singleprobe analysis (SPM). We have made the first these codes used for the likelihood estimation public. ${ }^{4}$

This work belongs to a group of papers published by the BOSS collaboration in an attempt to thoroughly study the data provided by its final data release. Baryon Acoustic Oscillations (BAO) in configuration and Fourier spaces was studied by Cuesta et al. (2016b), Gil-Marín et al. (2016b) and Beutler et al. (2017a). Gil-Marín et al. (2016a) and Beutler et al. (2017b) extended its study in Fourier space to full redshift space distortions by means of the power spectrum while Satpathy et al. (2016) did so in configuration space with the correlation function. Chuang et al. (2016a,b) used DR12 clustering as a 'single-probe' constraint adopting only broad priors in place of external data. Three-point correlation function has been considered too, see for example Slepian et al. (2016) and Slepian \& Eisenstein (2016). Grieb et al. (2017) and Sanchez et al. (2017b) divided the sample in wedges extracting their cosmological implications in Fourier and configuration space. Sanchez et al. (2017a) showed how to consistently combine measurements from several groups. A test over systematics was performed by Ross et al. (2017) and Vargas-Magaña et al. (2016). Salazar-Albornoz et al. (2016), Zhao et al. (2017) and Wang et al. (2016) worked on clustering tomography from three different perspectives (including Fourier and configuration spaces). This paper consistently includes CMB information and gives some clues about its relation with LSS. We refer to the 'consensus paper', Alam et al. (2016), for extra details on where this paper fits in the bigger picture.

This paper is organized as follows. In Section 2, we introduce the Planck data, the SDSS-III/BOSS DR12 galaxy sample and mock catalogues used in our study. In Section 3, we describe the details of the methodology that constrains cosmological parameters from our joint $\mathrm{CMB}$ and galaxy clustering analysis. In Section 4 , we present our double-probe cosmological measurements. In Section 5, we demonstrate how to derive cosmological constraints from our measurements with a given dark energy model. In Section 6, in contrast to the manner of dark energy model-independent method, we present the results from the full-likelihood analysis with fixed dark energy models. In Section 7, we measure the sum of the neutrino mass with different methodologies. We summarize and conclude in Section 8 .

\section{DATA SETS AND MOCKS}

\subsection{The SDSS-III/BOSS galaxy catalogues}

The SDSS survey (Fukugita et al. 1996; Gunn et al. 1998; York et al. 2000; Smee et al. 2013) mapped over a quarter of the sky using the dedicated $2.5 \mathrm{~m}$ Sloan Telescope (Gunn et al. 2006). The BOSS survey (Eisenstein et al. 2011; Bolton et al. 2012; Dawson et al. 2013) is part of the SDSS-III survey. It collects spectra and redshifts from 1.5 million galaxies, 160000 quasars and 100000 ancillary targets. Data Release 12 (Alam et al. 2015a) has already been made publicly available. ${ }^{5}$ We use galaxies from the SDSS-III BOSS DR12 CMASS catalogue in the redshift range $0.43<z<0.75$ and LOWZ catalogue in the range $0.15<z<0.43$. CMASS samples are selected with an approximately constant stellar mass threshold (Eisenstein et al. 2011), whereas the LOWZ sample consists of red galaxies at $z<0.4$ from the SDSS DR8 (Aihara et al. 2011) image data. We are using 800853 CMASS galaxies and 361775 LOWZ galaxies. The effective redshifts of the sample are $z=0.59$ and 0.32 , respectively. The details of generating this sample are described in Reid et al. (2016). One can check the data points used in this work in Fig. 1.

\subsection{The Planck data}

Planck (Tauber et al. 2010; Planck Collaboration I 2011) is a third-generation cosmological space mission, following $C O B E$ and Wilkinson Microwave Anisotropy Probe, to measure the anisotropy of the CMB. It observed the sky in nine frequency bands covering the range $30-857 \mathrm{GHz}$ with high sensitivity and angular resolutions from 31 to 5 arcmin. The Low Frequency Instrument (Bersanelli et al. 2010; Mennella et al. 2011) covers the bands centred at 30, 44 and $70 \mathrm{GHz}$ using pseudo-correlation radiometer detectors, while the High Frequency Instrument (HFI; Planck HFI Core Team 2011) covers the 100, 143, 217, 353, 545 and $857 \mathrm{GHz}$ bands with bolometers. Polarization is measured in all but the highest two bands (Leahy et al. 2010; Rosset et al. 2010). In this paper, we used the Planck 2015 release (Planck Collaboration I 2016a), which includes the full mission maps and associated data products. One can check the data points used in this work in Fig. 2.

\subsection{The mock galaxy catalogues}

We use 2000 BOSS DR12 MultiDark-PATCHY (MD-PATCHY) mock galaxy catalogues (Kitaura et al. 2016b) for validating our methodology and estimating the covariance matrix in this study. These mock catalogues were constructed using a similar procedure to that described in Rodríguez-Torres et al. (2016), who constructed the BOSS DR12 light-cone mock catalogues using the MultiDark $N$-body simulations. However, instead of using $N$-body simulations,

\footnotetext{
${ }^{4}$ http://www.iac.es/proyecto/lss/pages/en/software.php
}

${ }^{5}$ http://www.sdss3.org/ 

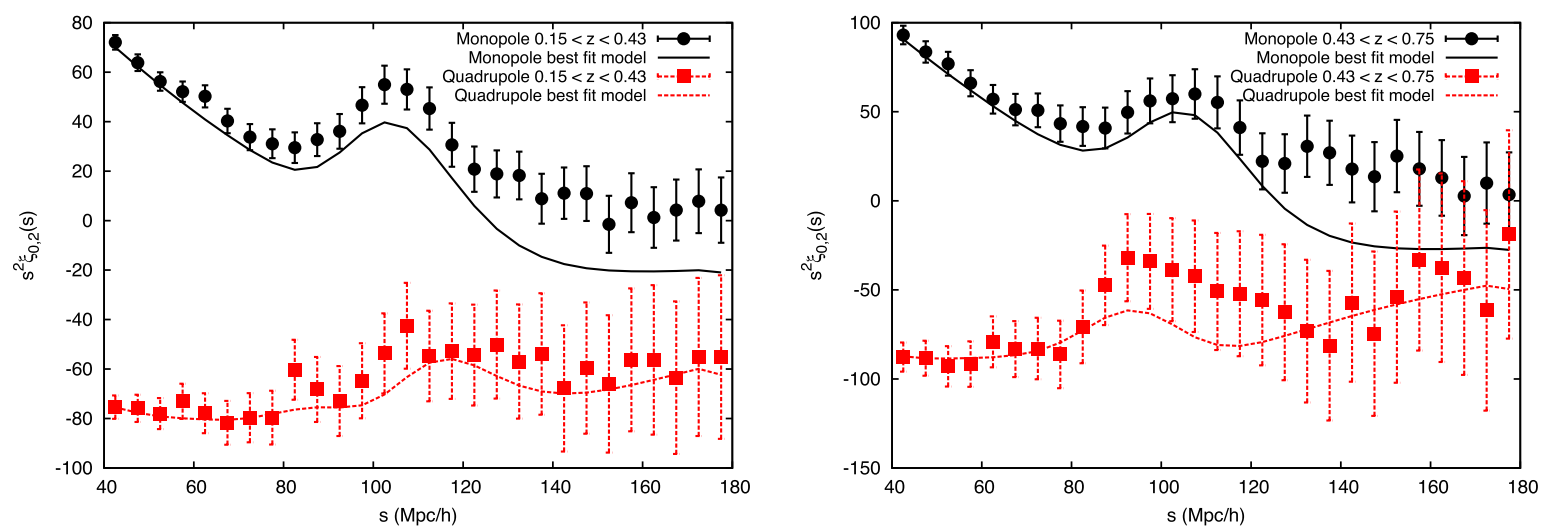

Figure 1. Left-hand panel: measurement of monopole and quadrupole of the correlation function from the BOSS DR12 LOWZ galaxy sample within $0.15<z<0.43$ compared to the best-fitting theoretical models (solid lines). Right-hand panel: measurement of effective monopole and quadrupole of the correlation function from the BOSS DR12 CMASS galaxy sample within $0.43<z<0.75$ compared to the best-fitting theoretical models (solid lines). The error bars are the square roots of the diagonal elements of the covariance matrix. In this study, our fitting scale ranges are $40 h^{-1} \mathrm{Mpc}<s<180 h^{-1} \mathrm{Mpc}$; the bin size is $5 h^{-1}$ Mpc. Two-point correlation functions from the same data were used by Cuesta et al. (2016b) and Sanchez et al. (2017b).

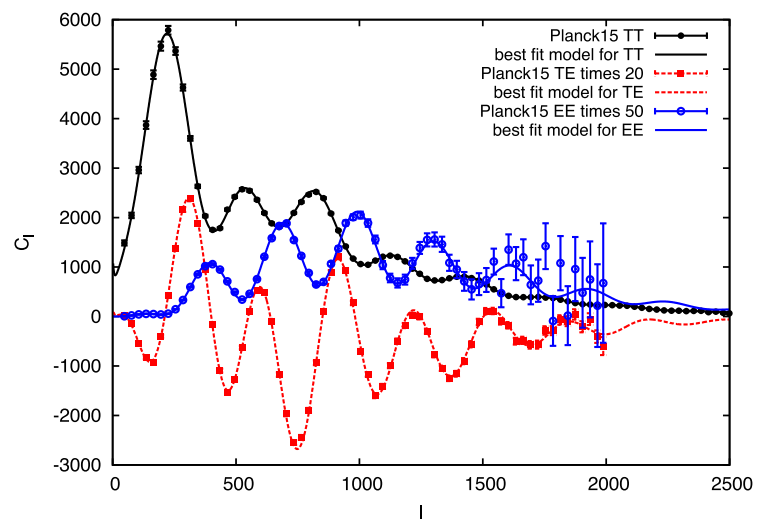

Figure 2. Angular power spectrum of temperature and polarization measurement from Planck data and their best fits from our double-probe analysis.

the 2000 MD-PATCHY mock catalogues were constructed using PATCHY approximate simulations. These mocks are produced using 10 boxes at different redshifts created with the PATCHY code (Kitaura, Yepes \& Prada 2014). The PATchY code consists of two stages: (1) computing approximate dark matter density field and (2) populating galaxies from dark matter density field with the biasing model. The dark matter density field is estimated using augmented Lagrangian perturbation theory (Kitaura \& Hess 2013), which combines the second-order perturbation theory (e.g. Buchert 1994; Bouchet et al. 1995; Catelan 1995) and the spherical collapse approximation (see Bernardeau 1994; Mohayaee et al. 2006; Neyrinck 2013). The biasing model includes deterministic bias and stochastic bias (see Kitaura et al. 2014, 2015 for details). The velocity field is constructed based on the displacement field of dark matter particles. Finger-of-god modelling has also been taken into account statistically. The mocks match the clustering of the galaxy catalogues for each redshift bin (see Kitaura et al. 2016b for details) and have been used in recent galaxy clustering studies (Cuesta et al. 2016b; Gil-Marín et al. 2016a,b; Rodríguez-Torres et al. 2016; Slepian et al. 2017) and void clustering studies (Kitaura et al. 2016a; Liang et al. 2016). They are also used in Alam et al. (2016, BOSS collaboration paper for final data release) and its companion papers [this paper and Ross et al. (2017), Vargas-Magaña et al. (2016), Beutler et al. (2017a,b), Satpathy et al. (2016), Sanchez et al. (2017a,b), Grieb et al. (2017), Chuang et al. (2016a), Slepian et al. (2016), Slepian \& Eisenstein (2016), Salazar-Albornoz et al. (2016), Zhao et al. (2017) and Wang et al. (2016)].

\section{METHODOLOGY}

We develop a new methodology to extract the cosmological constraints from the joint data set of the Planck CMB data and BOSS galaxy clustering measurements. We fit the LSS data using derived parameters that are combinations of basic parameters defining the key cosmological dependences $\left[H(z), D_{\mathrm{A}}(z)\right.$ and $\left.f(z) \sigma_{8}(z)\right]$, while including $\mathrm{CMB}$ data to simultaneously constrain other parameters $\left[\Omega_{\mathrm{c}} h^{2}, \Omega_{\mathrm{b}} h^{2}, n_{\mathrm{s}}, \log \left(A_{\mathrm{s}}\right), \theta\right.$ and $\left.\tau\right]$ in a model-independent manner. This means that we can define a parameter space that can subsequently be used to constrain a wide range of dark energy models. Similar approaches have been applied to these data separately. Our work is the first to investigate how in detail this joint analysis should be performed.

\subsection{Likelihood from BOSS galaxy clustering}

In this section, we describe the steps to compute the likelihood from the BOSS galaxy clustering.

\subsubsection{Measure multipoles of the two-point correlation function}

We convert the measured redshifts of the BOSS CMASS and LOWZ galaxies to comoving distances by assuming a fiducial model, i.e. flat $\Lambda$ cold dark matter $(\Lambda \mathrm{CDM})$ with $\Omega_{\mathrm{m}}=0.307115$ and $h=0.6777$, which is the same model adopted for constructing the mock catalogues (see Kitaura et al. 2016b). In order to compute the two-dimensional two-point correlation function, we use the two-point correlation function estimator given by Landy \& Szalay (1993):

$\xi(s, \mu)=\frac{\mathrm{DD}(s, \mu)-2 \mathrm{DR}(s, \mu)+\mathrm{RR}(s, \mu)}{\operatorname{RR}(s, \mu)}$,

where $s$ is the separation of a pair of objects and $\mu$ is the cosine of the angle between the line of sight (LOS) and the line connecting the pair of objects. DD, DR and RR represent the normalized datadata, data-random and random-random pair counts, respectively, for a given distance range. The LOS is defined as the direction 
Table 1. Fiducial result of the double-probe approach. The units of $H(z)$ and $D_{\mathrm{A}}(z)$ are $\mathrm{km} \mathrm{s}^{-1} \mathrm{Mpc}^{-1}$ and Mpc.

\begin{tabular}{lc}
\hline Parameter & Measurement \\
\hline$f \sigma_{8}(0.59)$ & $0.510 \pm 0.047$ \\
$H(0.59) r_{\mathrm{s}} / r_{\mathrm{s}, \text { fid }}$ & $97.9 \pm 3.1$ \\
$D_{\mathrm{A}}(0.59) r_{\mathrm{s}, \text { fid }} / r_{\mathrm{s}}$ & $1422 \pm 25$ \\
$f \sigma_{8}(0.32)$ & $0.431 \pm 0.063$ \\
$H(0.32) r_{\mathrm{s}} / r_{\mathrm{s}, \text { fid }}$ & $79.1 \pm 3.3$ \\
$D_{\mathrm{A}}(0.32) r_{\mathrm{s}, \text { fid }} / r_{\mathrm{s}}$ & $956 \pm 27$ \\
$R$ & $1.7430 \pm 0.0080$ \\
$l_{\mathrm{a}}$ & $301.70 \pm 0.15$ \\
$\Omega_{\mathrm{b}} h^{2}$ & $0.02233 \pm 0.00025$ \\
$n_{\mathrm{s}}$ & $0.9690 \pm 0.0066$ \\
$\ln \left(10^{10} A_{\mathrm{s}}\right)$ & $3.040 \pm 0.036$ \\
$\Omega_{\mathrm{k}}$ & $-0.003 \pm 0.006$ \\
\hline
\end{tabular}

from the observer to the centre of a galaxy pair. Our bin size is $\Delta s=1 h^{-1} \mathrm{Mpc}$ and $\Delta \mu=0.01$. The Landy and Szalay estimator has minimal variance for a Poisson process. Random data are generated with the same radial and angular selection functions as the real data. One can reduce the shot noise due to random data by increasing the amount of random data. The number of random data we use is about 50 times that of the real data. While calculating the pair counts, we assign to each data point a radial weight of $1 /\left[1+n(z) \cdot P_{w}\right]$, where $n(z)$ is the radial number density and $P_{w}=1 \times 10^{4} h^{-3} \mathrm{Mpc}^{3}$ (see Feldman, Kaiser \& Peacock 1994).

The traditional multipoles of the two-point correlation function, in redshift space, are defined by

$\xi_{l}(s) \equiv \frac{2 l+1}{2} \int_{-1}^{1} \mathrm{~d} \mu \xi(s, \mu) P_{l}(\mu)$,

where $P_{l}(\mu)$ is the Legendre polynomial ( $l=0$ and 2 here). We integrate over a spherical shell with radius $s$, while actual measurements of $\xi(s, \mu)$ are done in discrete bins. To compare the measured $\xi(s, \mu)$ and our theoretical model, the last integral in equation (2) should be converted into a sum,

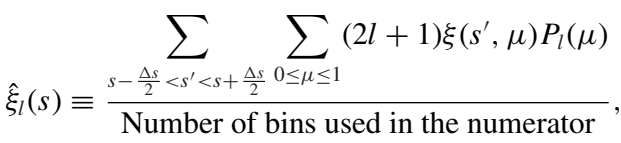

where $\Delta s=5 h^{-1} \mathrm{Mpc}$ in this work.

Fig. 1 shows the monopole $\left(\hat{\xi}_{0}\right)$ and quadrupole $\left(\hat{\xi}_{2}\right)$ measured from the BOSS CMASS and LOWZ galaxy sample compared with the best-fitting theoretical models without accounting for observational systematics in the measurement.
We are using the scale range $s=40-180 h^{-1} \mathrm{Mpc}$ so that we remain in the pseudo-linear regime of growth of structure, and the bin size is $5 h^{-1} \mathrm{Mpc}$. We define the vector of points from the multipoles in the scale range considered

$\boldsymbol{X}=\left\{\hat{\xi}_{0}^{(1)}, \hat{\xi}_{0}^{(2)}, \ldots, \hat{\xi}_{0}^{(N)} ; \hat{\xi}_{2}^{(1)}, \hat{\xi}_{2}^{(2)}, \ldots, \hat{\xi}_{2}^{(N)} ; \ldots\right\}$,

where $N$ is the number of data points in each measured multipole; here $N=28$. The length of the data vector $\boldsymbol{X}$ depends on the number of multipoles used.

\subsubsection{Theoretical two-point correlation function}

Following SPM, we use two models to compute the likelihood of the galaxy clustering measurements. One is a fast model used to narrow down the parameter space scanned; the other is a slower model used to calibrate the results from the fast model.

(i) Fast model. The fast model we use is the two-dimensional dewiggle model explained in SPM. The theoretical model can be constructed by first- and higher-order perturbation theory. We first adopt the CDM model and adiabatic initial conditions. Computing the linear matter power spectra, $P_{\operatorname{lin}}(k)$, by using CAMB (Code for Anisotropies in the Microwave Background; Lewis, Challinor \& Lasenby 2000), we can decompose it into two parts:

$P_{\text {lin }}(k)=P_{\text {nw }}(k)+P_{\mathrm{BAO}}^{\operatorname{lin}}(k)$,

where $P_{\mathrm{nw}}(k)$ is the 'no-wiggle' power spectrum calculated using equation (29) from Eisenstein \& $\mathrm{Hu}(1998)$ and $P_{\mathrm{BAO}}^{\text {lin }}(k)$ is the 'wiggled' part defined by previous equation (5). The non-linear damping effect of the 'wiggled' part, in redshift space, is approximated following Eisenstein, Seo \& White (2007) by

$P_{\mathrm{BAO}}^{\mathrm{nl}}\left(k, \mu_{k}\right)=P_{\mathrm{BAO}}^{\operatorname{lin}}(k) \cdot \exp \left(-\frac{k^{2}}{2 k_{\star}^{2}}\left[1+\mu_{k}^{2}\left(2 f+f^{2}\right)\right]\right)$,

where $\mu_{k}$ is the cosine of the angle between $\boldsymbol{k}$ and the LOS, $f$ is the growth rate, and $k_{\star}$ is computed following Crocce \& Scoccimarro (2006) and Matsubara (2008) by

$k_{\star}=\left[\frac{1}{3 \pi^{2}} \int P_{\text {lin }}(k) \mathrm{d} k\right]^{-1 / 2}$.

Thus, the dewiggled power spectrum is

$P_{\mathrm{dw}}\left(k, \mu_{k}\right)=P_{\mathrm{nw}}(k)+P_{\mathrm{BAO}}^{\mathrm{nl}}\left(k, \mu_{k}\right)$.

We include the linear redshift distortion as follows (Kaiser 1987),

$P_{\mathrm{g}}^{s}\left(k, \mu_{k}\right)=b^{2}\left(1+\beta \mu_{k}^{2}\right)^{2} P_{\mathrm{dw}}\left(k, \mu_{k}\right)$,

Table 2. Normalized covariance matrix of the fiducial result from the double-probe approach

\begin{tabular}{|c|c|c|c|c|c|c|c|c|c|c|c|c|}
\hline & $R$ & $l_{\mathrm{a}}$ & $\Omega_{\mathrm{b}} h^{2}$ & $n_{\mathrm{s}}$ & $\ln \left(10^{10} A_{\mathrm{s}}\right)$ & $f \sigma_{8}(0.59)$ & $\frac{H(0.59)}{r_{\mathrm{s}, \mathrm{fid}} / r_{\mathrm{s}}}$ & $\frac{D_{\mathrm{A}}(0.59)}{r_{\mathrm{s}} / r_{\mathrm{s}, \text { fid }}}$ & $f \sigma_{8}(0.32)$ & $\frac{H(0.32)}{r_{\mathrm{s}, \mathrm{fid}} / r_{\mathrm{s}}}$ & $\frac{D_{\mathrm{A}}(0.32)}{r_{\mathrm{s}} / r_{\mathrm{s}, \mathrm{fid}}}$ & $\Omega_{\mathrm{k}}$ \\
\hline$l_{\mathrm{a}}$ & 0.6534 & 1.0000 & -0.5212 & -0.5770 & -0.0651 & -0.1067 & -0.1957 & 0.0017 & 0.0073 & 0.0174 & -0.0211 & 0.4329 \\
\hline$n_{\mathrm{s}}$ & -0.8787 & -0.5770 & 0.6633 & 1.0000 & 0.0808 & 0.0381 & 0.1648 & -0.0003 & 0.0285 & 0.0510 & 0.0303 & -0.5547 \\
\hline $\ln \left(10^{10} A_{\mathrm{s}}\right)$ & -0.0352 & -0.0651 & 0.1175 & 0.0808 & 1.0000 & 0.0034 & 0.0391 & 0.0175 & -0.0066 & 0.0020 & 0.0516 & 0.5915 \\
\hline$f \sigma_{8}(0.59)$ & -0.0620 & -0.1067 & 0.0525 & 0.0381 & 0.0034 & 1.0000 & 0.7153 & 0.6172 & 0.1531 & 0.1535 & -0.0333 & 0.0252 \\
\hline$f \sigma_{8}(0.32)$ & -0.0237 & 0.0073 & 0.1373 & 0.0285 & -0.0066 & 0.1531 & 0.0447 & 0.0209 & 1.0000 & 0.6581 & 0.5250 & 0.1142 \\
\hline$H(0.32) r_{\mathrm{s}} / r_{\mathrm{s}, \text { fid }}$ & -0.0271 & 0.0174 & 0.0566 & 0.0510 & 0.0020 & 0.1535 & 0.0968 & -0.0319 & 0.6581 & 1.0000 & 0.3168 & 0.1165 \\
\hline$D_{\mathrm{A}}(0.32) r_{\mathrm{s}, \text { fid }} / r_{\mathrm{s}}$ & 0.0027 & -0.0211 & 0.0321 & 0.0303 & 0.0516 & -0.0333 & -0.0388 & -0.0839 & 0.5250 & 0.3168 & 1.0000 & 0.0835 \\
\hline$\Omega_{\mathrm{k}}$ & 0.6349 & 0.4329 & -0.4070 & -0.5547 & 0.5915 & 0.0252 & -0.0959 & 0.0038 & 0.1142 & 0.1165 & 0.0835 & 1.0000 \\
\hline
\end{tabular}


Table 3. Constraints on cosmological parameters obtained by using our results assuming dark energy models (see Section 5).

\begin{tabular}{lcccccc}
\hline & $\Omega_{\mathrm{m}}$ & $H_{0}$ & $\sigma_{8}$ & $\Omega_{\mathrm{k}}$ & $w$ or $w_{0}$ & $w_{\mathrm{a}}$ \\
\hline$\Lambda \mathrm{CDM}$ & $0.304 \pm 0.009$ & $68.2 \pm 0.7$ & $0.806 \pm 0.014$ & 0 & -1 & 0 \\
$\mathrm{o} \Lambda \mathrm{CDM}$ & $0.303 \pm 0.010$ & $68.6 \pm 0.9$ & $0.810 \pm 0.015$ & $0.002 \pm 0.003$ & -1 & 0 \\
$w \mathrm{CDM}$ & $0.299 \pm 0.013$ & $69.0 \pm 1.5$ & $0.815 \pm 0.020$ & 0 & $-1.04 \pm 0.06$ & 0 \\
o $w \mathrm{CDM}$ & $0.302 \pm 0.014$ & $68.7 \pm 1.5$ & $0.811 \pm 0.021$ & $0.002 \pm 0.003$ & $-1.00 \pm 0.07$ & 0 \\
$w_{0} w_{\mathrm{a}} \mathrm{CDM}$ & $0.313 \pm 0.020$ & $67.6 \pm 2.0$ & $0.817 \pm 0.016$ & 0 & $-0.84 \pm 0.22$ & $-0.66 \pm 0.68$ \\
$\mathrm{o} w_{0} w_{\mathrm{a}} \mathrm{CDM}$ & $0.313 \pm 0.020$ & $67.6 \pm 2.2$ & $0.815 \pm 0.016$ & $0.000 \pm 0.004$ & $-0.85 \pm 0.24$ & $-0.61 \pm 0.80$ \\
\hline
\end{tabular}

Table 4. Constraints on cosmological parameters from full-likelihood MCMC analysis of the joint data set (see Section 6).

\begin{tabular}{lcccccc}
\hline & $\Omega_{\mathrm{m}}$ & $H_{0}$ & $\sigma_{8}$ & $\Omega_{\mathrm{k}}$ & $w$ or $w_{0}$ & $w_{\mathrm{a}}$ \\
\hline$\Lambda \mathrm{CDM}$ & $0.305 \pm 0.008$ & $68.0 \pm 0.6$ & $0.812 \pm 0.009$ & 0 & -1 & 0 \\
$\mathrm{o} \Lambda \mathrm{CDM}$ & $0.300 \pm 0.009$ & $68.6 \pm 1.0$ & $0.816 \pm 0.010$ & $0.001 \pm 0.003$ & -1 & 0 \\
$w \mathrm{CDM}$ & $0.298 \pm 0.015$ & $68.8 \pm 1.8$ & $0.818 \pm 0.017$ & 0 & $-1.02 \pm 0.07$ & 0 \\
$\mathrm{o} w \mathrm{CDM}$ & $0.298 \pm 0.017$ & $68.8 \pm 1.8$ & $0.818 \pm 0.018$ & $0.001 \pm 0.003$ & $-1.01 \pm 0.08$ & 0 \\
$w_{0} w_{\mathrm{a}} \mathrm{CDM}$ & $0.311 \pm 0.022$ & $67.4 \pm 2.3$ & $0.808 \pm 0.020$ & 0 & $-0.85 \pm 0.23$ & $-0.51 \pm 0.67$ \\
$\mathrm{o} w_{0} w_{\mathrm{a}} \mathrm{CDM}$ & $0.309 \pm 0.025$ & $67.8 \pm 3.0$ & $0.810 \pm 0.024$ & $0.000 \pm 0.004$ & $-0.86 \pm 0.26$ & $-0.50 \pm 0.73$ \\
\hline
\end{tabular}

where $b$ is the linear galaxy bias and $\beta$ is the linear redshift distortion parameter.

To compute the theoretical two-point correlation function, $\xi(s, \mu)$, we Fourier transform the non-linear power spectrum $P_{\mathrm{g}}^{s}\left(k, \mu_{k}\right)$ by using Legendre polynomial expansions and one-dimensional integral convolutions as introduced in Chuang \& Wang (2013b).

We multiply the fast model multipoles by two functions depending on the distance $s$

$\xi_{0}^{\mathrm{cal}}(s)=\left(1-\mathrm{e}^{-\frac{s}{s_{1}}}+\mathrm{e}^{-\left(\frac{s}{s_{2}}\right)^{2}}\right) \xi_{0}(s)$,

$\xi_{2}^{\mathrm{cal}}(s)=\left(1-\mathrm{e}^{-\frac{s}{s_{3}}}+\mathrm{e}^{-\left(\frac{s}{s_{4}}\right)^{2}}\right) \xi_{2}(s)$,

so that it mimics the slow model presented below. We find the calibration parameters, $s_{1}=12, s_{2}=14, s_{3}=20$ and $s_{4}=27$, by comparing the fast and slow models. It is not critical to find the best form of calibration function and its parameters as the model will be calibrated in the next step when performing importance sampling with slow model.

(ii) Slow model. The slower but more accurate model we use is the 'Gaussian streaming model' described in Reid \& White (2011). The model assumes that the pairwise velocity probability distribution function is Gaussian and can be used to relate real space clustering and pairwise velocity statistics of haloes to their clustering in redshift space by

$$
\begin{aligned}
1+ & \xi_{\mathrm{g}}^{s}\left(r_{\sigma}, r_{\pi}\right) \\
& =\int\left[1+\xi_{\mathrm{g}}^{r}(r)\right] \mathrm{e}^{-\left[r_{\pi}-y-\mu v_{12}(r)\right]^{2} / 2 \sigma_{12}^{2}(r, \mu)} \frac{\mathrm{d} y}{\sqrt{2 \pi \sigma_{12}^{2}(r, \mu)}},
\end{aligned}
$$

where $r_{\sigma}$ and $r_{\pi}$ are the redshift space transverse and LOS distances between two objects with respect to the observer, $y$ is the real space LOS pair separation, $\mu=y / r, \xi_{\mathrm{g}}^{\mathrm{r}}$ is the real space galaxy correlation function, $v_{12}(r)$ is the average infall velocity of galaxies separated by real space distance $r$ and $\sigma_{12}^{2}(r, \mu)$ is the rms dispersion of the pairwise velocity between two galaxies separated with transverse (LOS) real space separation $r_{\sigma}(y) . \xi_{\mathrm{g}}^{\mathrm{r}}(r), v_{12}(r)$ and $\sigma_{12}^{2}(r, \mu)$ are computed in the framework of Lagrangian $\left(\xi^{\mathrm{r}}\right)$ and standard perturbation theories $\left(v_{12}, \sigma_{12}^{2}\right)$.

For large scales, only one nuisance parameter is necessary to describe the clustering of a sample of haloes or galaxies in this model: $b_{1 L}=b-1$, the first-order Lagrangian host halo bias in real space.

In this study, we consider relative large scales (i.e. $40<s<180 h^{-1} \mathrm{Mpc}$ ), so that we do not include $\sigma_{\mathrm{FoG}}^{2}$, to model a velocity dispersion accounting for small-scale motions of haloes and galaxies. Further details of the model, its numerical implementation and its accuracy can be found in Reid \& White (2011).

\subsubsection{Covariance matrix}

We use the 2000 mock catalogues created by Kitaura et al. (2016b) for the BOSS DR12 CMASS and LOWZ galaxy sample to estimate the covariance matrix of the observed correlation function. We calculate the multipoles of the correlation functions of the mock catalogues and construct the covariance matrix as

$C_{i j}=\frac{1}{(N-1)(1-D)} \sum_{k=1}^{N}\left(\bar{X}_{i}-X_{i}^{k}\right)\left(\bar{X}_{j}-X_{j}^{k}\right)$,

where

$D=\frac{N_{\mathrm{b}}+1}{N-1}$,

$N$ is the number of the mock catalogues, $N_{\mathrm{b}}$ is the number of data bins, $\bar{X}_{m}$ is the mean of the $m$ th element of the vector from the mock catalogue multipoles and $X_{m}^{k}$ is the value in the $m$ th elements of the vector from the $k$ th mock catalogue multipoles. The vector $\boldsymbol{X}$ is defined by equation (4). We also include the correction, $D$, introduced by Hartlap, Simon \& Schneider (2007).

\subsubsection{Compute likelihood from galaxy clustering}

The likelihood is taken to be proportional to $\exp \left(-\chi^{2} / 2\right)$, with $\chi^{2}$ given by

$\chi^{2} \equiv \sum_{i, j=1}^{N_{X}}\left[X_{\mathrm{th}, i}-X_{\mathrm{obs}, i}\right] C_{i j}^{-1}\left[X_{\mathrm{th}, j}-X_{\mathrm{obs}, j}\right]$,

where $N_{X}$ is the length of the vector used, $X_{\mathrm{th}}$ is the vector from the theoretical model and $X_{\mathrm{obs}}$ is the vector from the observed data.

As explained in Chuang \& Wang (2012), instead of recalculating the observed correlation function while computing for different 

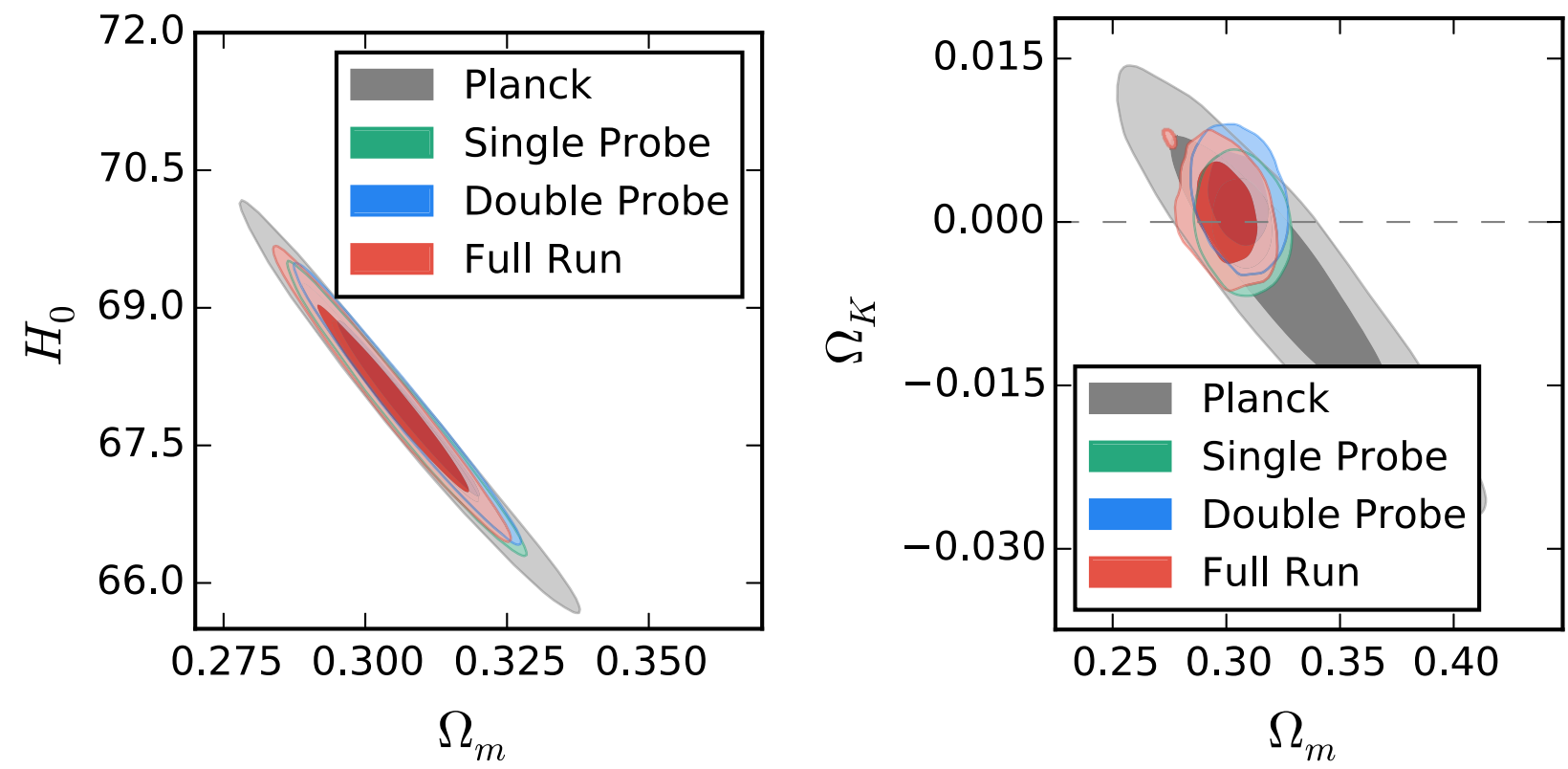

Figure 3. Left-hand panel: 2D marginalized contours for 68 and 95 per cent confidence levels for $\Omega_{\mathrm{m}}$ and $H_{0}$ ( $\Lambda$ CDM model assumed) from Planck-only (grey), derived using double-probe measurements (blue), full-likelihood analysis with joint data (red; labelled as 'full run') and Planck+single-probe measurements (green). Right-hand panel: 2D marginalized contours for 68 and 95 per cent confidence level for $\Omega_{\mathrm{m}}$ and $\Omega_{\mathrm{k}}$ (o $\Lambda \mathrm{CDM}$ model assumed). One can see that the latter three measurements are consistent with each other.
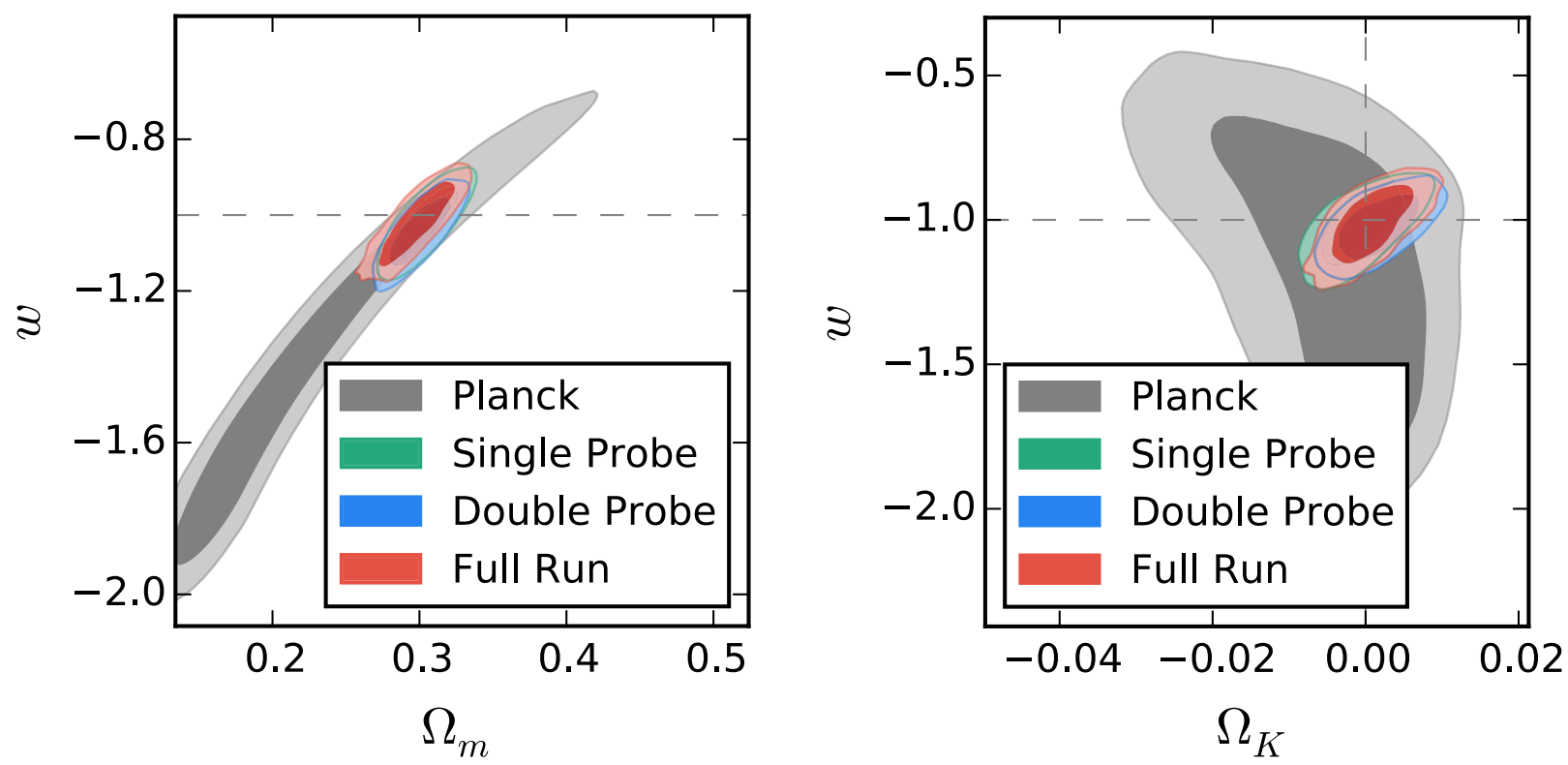

Figure 4. Left-hand panel: 2D marginalized contours for 68 and 95 per cent confidence level for $\Omega_{\mathrm{m}}$ and $w$ ( $w$ CDM model assumed) from Planck-only (grey), derived using double-probe measurements (blue), full-likelihood analysis with joint data (red; labelled as 'full run') and Planck+single-probe measurements (green). Right-hand panel: 2D marginalized contours for 68 and 95 per cent confidence level for $\Omega_{\mathrm{k}}$ and $w$ (owCDM model assumed). One can see that the latter three measurements are consistent with each other.

models, we rescale the theoretical correlation function to avoid rendering the $\chi^{2}$ values arbitrary. This approach can be considered as an application of Alcock-Paczynski effect (Alcock \& Paczynski 1979). The rescaled theoretical correlation function is computed by

$$
T^{-1}\left(\xi_{\mathrm{th}}(\sigma, \pi)\right)=\xi_{\mathrm{th}}\left(\frac{D_{\mathrm{A}}(z)}{D_{\mathrm{A}}^{\mathrm{fid}}(z)} \sigma, \frac{H^{\mathrm{fid}}(z)}{H(z)} \pi\right),
$$

where $\xi_{\text {th }}$ is the theoretical model computed in Section 3.1.2. $T^{-1}$ is the function in charge of rescaling distances. Here, $D_{\mathrm{A}}(z)$ and $H(z)$ would be the input parameters, and $D_{\mathrm{A}}^{\mathrm{fid}}(z)$ and $H^{\mathrm{fid}}(z)$ are $\left\{990.20 \mathrm{Mpc}, 80.16 \mathrm{~km} \mathrm{~s}^{-1} \mathrm{Mpc}^{-1}\right\}$ at $z=0.32$ (LOWZ) and $\left\{1409.26 \mathrm{Mpc}, 94.09 \mathrm{~km} \mathrm{~s}^{-1} \mathrm{Mpc}^{-1}\right\}$ at $z=0.59$ (CMASS). Then, $\chi^{2}$ can be rewritten as

$$
\begin{aligned}
\chi^{2} \equiv & \sum_{i, j=1}^{N_{X}}\left\{T^{-1} X_{\mathrm{th}, i}-X_{\mathrm{obs}, i}^{\mathrm{fid}}\right\} C_{\mathrm{fid}, i j}^{-1} . \\
& \cdot\left\{T^{-1} X_{\mathrm{th}, j}-X_{\mathrm{obs}, j}^{\mathrm{fid}}\right\} ;
\end{aligned}
$$



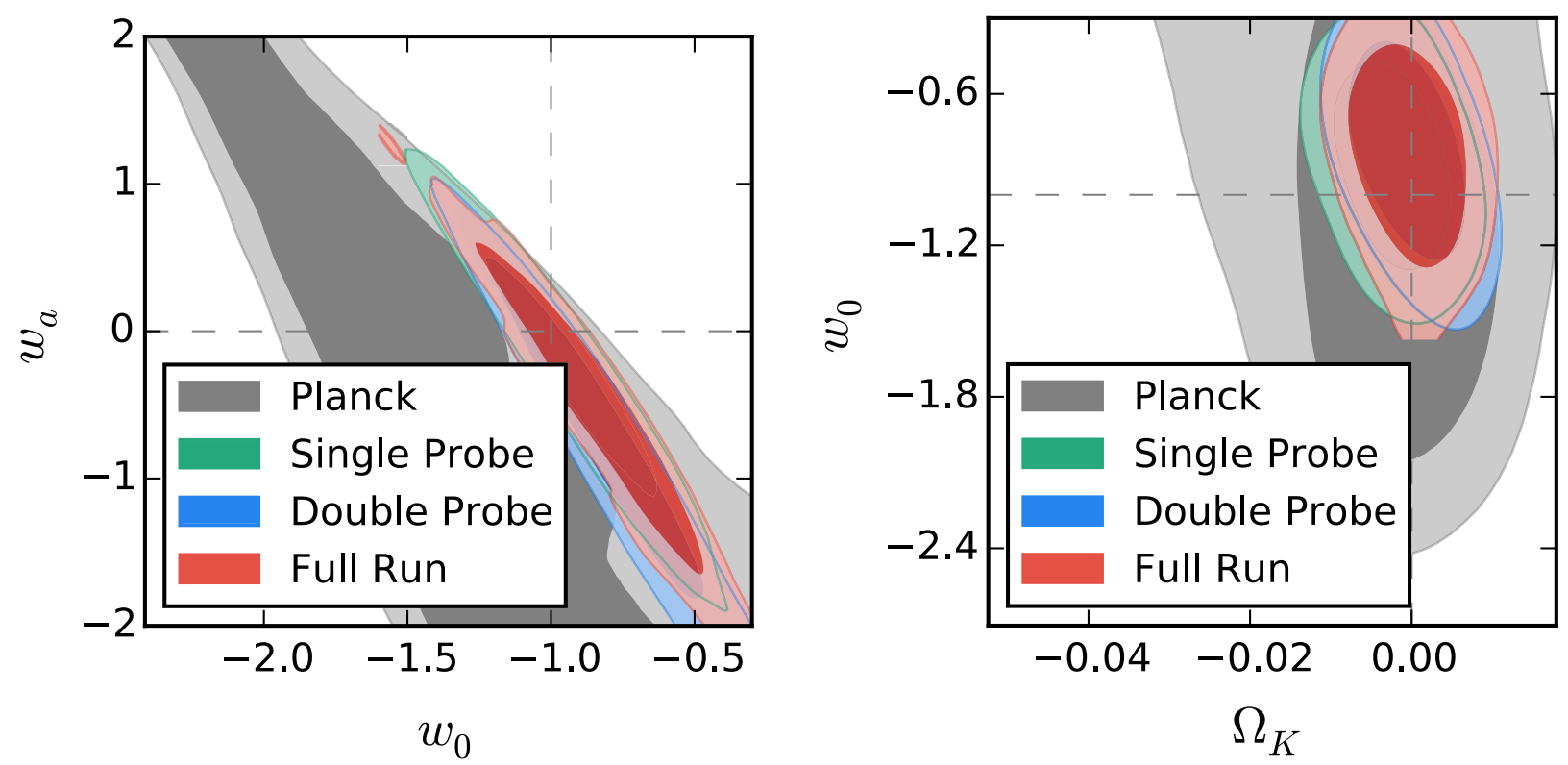

Figure 5. Left-hand panel: 2D marginalized contours for 68 and 95 percent confidence level for $w_{0}$ and $w_{\mathrm{a}}$ ( $w_{0} w_{\mathrm{a}} \mathrm{CDM}$ model assumed) from Planckonly (grey), derived using double-probe measurements (blue), full-likelihood analysis with joint data (red; labelled as 'full run') and Planck+single-probe measurements (green). Right-hand panel: 2D marginalized contours for 68 and 95 per cent confidence level for $\Omega_{\mathrm{k}}$ and $w_{0}$ (ow $w_{0} w_{\mathrm{a}} \mathrm{CDM}$ model assumed). One can see that the latter three measurements are consistent with each other.

where $T^{-1} X_{\text {th }}$ is the vector computed by equation (3) from the rescaled theoretical correlation function, equation (16). $X_{\text {obs }}^{\text {fid }}$ is the vector from observed data measured with the fiducial model (see Chuang \& Wang 2012 for more details regarding the rescaling method).

\subsection{Likelihood from Planck CMB data}

Our CMB data set consists of the Planck 2015 measurements (Planck Collaboration I 2016a; Planck Collaboration XIII 2016c). The reference likelihood code (Planck Collaboration XI 2016b) was downloaded from the Planck Legacy Archive. ${ }^{6}$ Here, we combine the Plik baseline likelihood for high multipoles $(30 \leq \ell \leq 2500)$ using the TT, TE and EE power spectra, and the Planck low- $\ell$ multipole likelihood in the range $2 \leq \ell \leq 29$ (hereafter lowTEB). We also include the new Planck 2015 lensing likelihood (Planck Collaboration XV 2016d), constructed from the measurements of the power spectrum of the lensing potential (hereafter referred as 'lensing'). Using the Planck lensing likelihood, the $A_{\text {lens }}$ parameter is always set to 1 (Planck Collaboration XIII 2016c).

\subsection{MCMC likelihood analysis}

\subsubsection{Basic procedure}

We perform MCMC likelihood analyses using соммомс (Lewis \& Bridle 2002; Lewis 2013). The fiducial parameter space that we explore spans the parameter set of $\left\{\Omega_{\mathrm{c}} h^{2}, \Omega_{\mathrm{b}} h^{2}, n_{\mathrm{s}}, \log \left(A_{\mathrm{s}}\right), \theta, \tau\right.$, $\left.\Omega_{\mathrm{k}}, w, H(z), D_{\mathrm{A}}(z), \beta(z), b \sigma_{8}(z), b(z)\right\}$. The quantities $\Omega_{\mathrm{c}}$ and $\Omega_{\mathrm{b}}$ are the CDM and baryon density fractions, $n_{\mathrm{s}}$ is the power-law index of the primordial matter power spectrum, $\Omega_{\mathrm{k}}$ is the curvature density fraction, $w$ is the equation of state of dark energy, $h$ is the dimensionless Hubble constant $\left(H_{0}=100 h \mathrm{~km} \mathrm{~s}^{-1} \mathrm{Mpc}^{-1}\right)$ and

${ }^{6}$ PLA: http://pla.esac.esa.int/. $\sigma_{8}(z)$ is the normalization of the power spectrum. Note that, with the joint data set (Planck + BOSS), the only parameter that is not well constrained is $b(z)$. We apply a flat prior of $(1,3)$ on it. The linear redshift distortion parameter can be expressed as $\beta(z)=f(z) / b$. Thus, one can derive $f(z) \sigma_{8}(z)$ from the measured $\beta(z)$ and $b \sigma_{8}(z)$.

\subsubsection{Generate Markov chains with fast model}

We first use the fast model (2D dewiggle model) to compute a first estimate of the likelihood, $\mathcal{L}_{\text {fast }}$, and generate the Markov chains. The Monte Carlo analysis will go through many random steps keeping or throwing the computed points' parameter space according to the Markov likelihood algorithm. Eventually, it will provide the chains of parameter points with high likelihood describing the constraints to our model.

\subsubsection{Calibrate the likelihood using slow model}

Once we have the fast model generated chains, we modify the weight of each point by

$\mathcal{W}_{\text {new }}=\mathcal{W}_{\text {old }} \frac{\mathcal{L}_{\text {slow }}}{\mathcal{L}_{\text {fast }}}$

where $\mathcal{L}_{\text {slow }}$ and $\mathcal{L}_{\text {fast }}$ are the likelihood for given point of input parameters in the chains. We save time by computing only the 'important' points without computing the likelihood of the ones that would not have been included in the first place. The methodology is known as 'importance sampling'. However, the typical importance sampling method is to add the likelihood of some additional data set to the given chains, but in this study, we replace the likelihood of a data set so that we do not count it twice.

\section{DOUBLE-PROBE RESULTS}

The two-point statistic of galaxy clustering cosmological information can be summarized by $\left\{\Omega_{\mathrm{m}} h^{2}, H(z), D_{\mathrm{A}}(z), f(z) \sigma_{8}(z)\right\}$ (e.g. 
Table 5. Results of double-probe analysis obtained with varying $\Sigma m_{v}$. The units of $H(z)$ and $D_{\mathrm{A}}(z)$ are $\mathrm{km} \mathrm{s}^{-1} \mathrm{Mpc}^{-1}$ and $\mathrm{Mpc}$ (see Section 7.1).

\begin{tabular}{lc}
\hline Parameter & Measurement \\
\hline$f \sigma_{8}(0.59)$ & $0.495 \pm 0.051$ \\
$H(0.59) r_{\mathrm{s}} / r_{\mathrm{s}, \text { fid }}$ & $97.5 \pm 3.2$ \\
$D_{\mathrm{A}}(0.59) r_{\mathrm{s}, \text { fid }} / r_{\mathrm{s}}$ & $1419 \pm 27$ \\
$f \sigma_{8}(0.32)$ & $0.431 \pm 0.066$ \\
$H(0.32) r_{\mathrm{s}} / r_{\mathrm{s}, \text { fid }}$ & $78.9 \pm 3.6$ \\
$D_{\mathrm{A}}(0.32) r_{\mathrm{s}, \text { fid }} / r_{\mathrm{s}}$ & $964 \pm 26$ \\
$\Omega_{\mathrm{bc}} h^{2}$ & $0.1413 \pm 0.0022$ \\
$l_{\mathrm{a}}$ & $301.75 \pm 0.14$ \\
$\Omega_{\mathrm{b}} h^{2}$ & $0.02209 \pm 0.00025$ \\
$n_{\mathrm{s}}$ & $0.9639 \pm 0.0068$ \\
$\ln \left(10^{10} A_{\mathrm{s}}\right)$ & $3.062 \pm 0.040$ \\
$\Omega_{\mathrm{k}}$ & $-0.009 \pm 0.006$ \\
\hline
\end{tabular}

Chuang \& Wang 2013b). By this statement, we mean that one can recover the same cosmological information using a full MCMC analysis of the two-point correlation function or power spectrum assuming a dark energy model or, alternatively, one can perform the measurement of those summarized parameters and then assume a dark energy model. In some studies, $\Omega_{\mathrm{m}} h^{2}$ was not included since a strong prior had been applied. Instead of using $H(z)$ and $D_{\mathrm{A}}(z)$, one uses the derived parameters $H(z) r_{\mathrm{s}} / r_{\mathrm{s} \text {, fid }}$ and $D_{\mathrm{A}}(z) r_{\mathrm{s}, \text { fid }} / r_{\mathrm{s}}$ to summarize the cosmological information since these two quantities are basically uncorrelated to $\Omega_{\mathrm{m}} h^{2}$, where $r_{\mathrm{s}}$ is the sound horizon at the redshift of the drag epoch and $r_{\mathrm{s} \text {, fid }}$ is the $r_{\mathrm{s}}$ of the fiducial cosmology. In this study, $\Omega_{\mathrm{m}} h^{2}$ is well constrained by the joint data set, but we still use $H(z) r_{\mathrm{s}} / r_{\mathrm{s} \text {, fid }}$ and $D_{\mathrm{A}}(z) r_{\mathrm{s} \text {, fid }} / r_{\mathrm{s}}$ because of their tighter constraints.

Wang \& Mukherjee (2007) showed that CMB shift parameters $\left(l_{\mathrm{a}}, R\right)$, together with $\Omega_{\mathrm{b}} h^{2}$, provide an efficient and intuitive

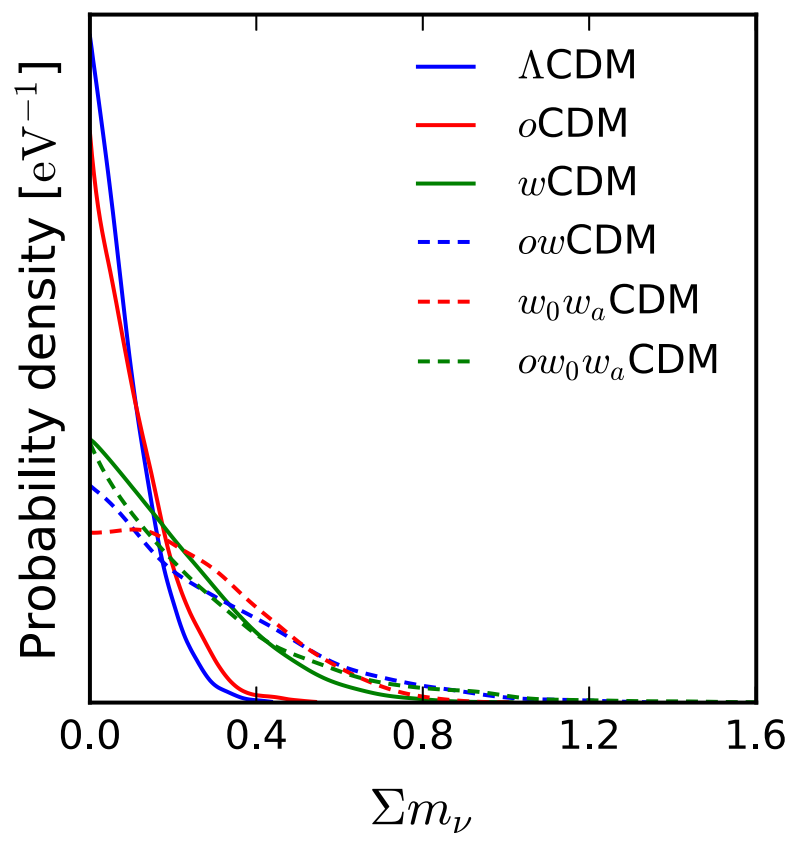

Figure 6. Probability density for $\Sigma m_{v}$ from double-probe measurements using the covariance matrix with free parameter $\Sigma m_{v}$ (see Section 7.1 and Table 7).

summary of CMB data as far as dark energy constraints are concerned. It is equivalent to replacing $\Omega_{\mathrm{b}} h^{2}$ with $z_{*}$, the redshift to the photon-decoupling surface (Wang 2009). The CMB shift parameters are defined as

$$
\begin{aligned}
& R \equiv \sqrt{\Omega_{\mathrm{m}} H_{0}^{2}} r\left(z_{*}\right), \\
& l_{\mathrm{a}} \equiv \pi r\left(z_{*}\right) / r_{\mathrm{s}}\left(z_{*}\right),
\end{aligned}
$$

\begin{tabular}{|c|c|c|c|c|c|c|c|c|c|c|c|c|}
\hline & $\Omega_{\mathrm{bc}} h^{2}$ & $l_{\mathrm{a}}$ & $\Omega_{\mathrm{b}} h^{2}$ & $n_{\mathrm{s}}$ & $\ln \left(10^{10} A_{\mathrm{s}}\right)$ & $f \sigma_{8}(0.59)$ & $\frac{H(0.59)}{r_{\mathrm{s}, \mathrm{fid}} / r_{\mathrm{s}}}$ & $\frac{D_{\mathrm{A}}(0.59)}{r_{\mathrm{s}} / r_{\mathrm{s}, \mathrm{fid}}}$ & $f \sigma_{8}(0.32)$ & $\frac{H(0.32)}{r_{\mathrm{s}, \mathrm{fid}} / r_{\mathrm{s}}}$ & $\frac{D_{\mathrm{A}}(0.32)}{r_{\mathrm{s}} / r_{\mathrm{s}, \mathrm{fid}}}$ & $\Omega_{\mathrm{k}}$ \\
\hline$l_{\mathrm{a}}$ & 0.4607 & 1.0000 & -0.4977 & -0.5042 & -0.0470 & 0.0201 & -0.0525 & 0.0043 & -0.0216 & 0.0765 & 0.0912 & 0.2919 \\
\hline$n_{\mathrm{s}}$ & -0.8376 & -0.5042 & 0.7188 & 1.0000 & 0.0475 & -0.0131 & -0.0591 & -0.0499 & 0.0717 & 0.0268 & -0.0686 & -0.2894 \\
\hline $\ln \left(10^{10} A_{\mathrm{s}}\right)$ & 0.0145 & -0.0470 & -0.0241 & 0.0475 & 1.0000 & 0.0095 & -0.0352 & -0.0065 & 0.0773 & 0.0225 & 0.0053 & 0.5576 \\
\hline$f \sigma_{8}(0.59)$ & 0.0075 & 0.0201 & -0.0016 & -0.0131 & 0.0095 & 1.0000 & 0.6546 & 0.5223 & 0.2427 & 0.2074 & 0.0634 & 0.1538 \\
\hline$f \sigma_{8}(0.32)$ & -0.0870 & -0.0216 & 0.0692 & 0.0717 & 0.0773 & 0.2427 & 0.0586 & -0.0598 & 1.0000 & 0.6531 & 0.4819 & 0.1487 \\
\hline$H(0.32) r_{\mathrm{s}} / r_{\mathrm{s}, \text { fid }}$ & 0.0317 & 0.0765 & 0.0299 & 0.0268 & 0.0225 & 0.2074 & 0.0615 & 0.0272 & 0.6531 & 1.0000 & 0.1686 & 0.1165 \\
\hline$D_{\mathrm{A}}(0.32) r_{\mathrm{s}, \text { fid }} / r_{\mathrm{s}}$ & 0.0049 & 0.0912 & 0.0149 & -0.0686 & 0.0053 & 0.0634 & 0.0015 & -0.0474 & 0.4819 & 0.1686 & 1.0000 & 0.0049 \\
\hline$\Omega_{\mathrm{k}}$ & 0.3794 & 0.2919 & -0.2708 & -0.2894 & 0.5576 & 0.1538 & -0.0025 & -0.0578 & 0.1487 & 0.1165 & 0.0049 & 1.0000 \\
\hline
\end{tabular}

Table 6. Correlation matrix of the double-probe measurements obtained with varying $\Sigma m_{v}$ (corresponding to Table 5; see Section 7.1).

Table 7. Constraints on cosmological parameters obtained by using the double-probe measurements presented in Tables 5 and 6 assuming dark energy models. We show 68 per cent 1D marginalized constraints for all the parameters. We provide also 95 per cent constraints for the neutrino masses in the parentheses.

\begin{tabular}{|c|c|c|c|c|c|c|c|}
\hline & $\Omega_{\mathrm{m}}$ & $H_{0}$ & $\sigma_{8}$ & $\Omega_{\mathrm{k}}$ & $w$ or $w_{0}$ & $w_{\mathrm{a}}$ & $\Sigma m_{\mu}(\mathrm{eV})$ \\
\hline $\mathrm{o} \Lambda \mathrm{CDM}$ & $0.310 \pm 0.011$ & $67.8 \pm 1.0$ & $0.828 \pm 0.020$ & $0.002 \pm 0.003$ & -1 & 0 & $<0.13(<0.27)$ \\
\hline owCDM & $0.297 \pm 0.017$ & $69.8 \pm 2.2$ & $0.816 \pm 0.033$ & $0.001 \pm 0.004$ & $-1.13 \pm 0.12$ & 0 & $<0.35(<0.75)$ \\
\hline$w_{0} w_{\mathrm{a}} \mathrm{CDM}$ & $0.312 \pm 0.024$ & $68.1 \pm 2.6$ & $0.812 \pm 0.030$ & 0 & $-0.88 \pm 0.24$ & $-0.89 \pm 0.75$ & $<0.32(<0.60)$ \\
\hline $\mathrm{o} w_{0} w_{\mathrm{a}} \mathrm{CDM}$ & $0.310 \pm 0.026$ & $68.3 \pm 3.3$ & $0.809 \pm 0.034$ & $-0.001 \pm 0.004$ & $-0.91 \pm 0.29$ & $-0.83 \pm 0.87$ & $<0.31(<0.78)$ \\
\hline
\end{tabular}
The units of $H_{0}$ and $\Sigma m_{v}$ are $\mathrm{km} \mathrm{s}^{-1} \mathrm{Mpc}^{-1}$ and eV, respectively (see Section 7.1 and Fig. 6). 
Table 8. Constraints on cosmological parameters obtained by using our double-probe measurements obtained with fixed $\Sigma m_{v}$ assuming dark energy models. We show 68 per cent 1D marginalized constraints for all the parameters. We provide also 95 per cent constraints for the neutrino masses in the parentheses. The units of $H_{0}$ and $\Sigma m_{v}$ are $\mathrm{km} \mathrm{s}^{-1} \mathrm{Mpc}^{-1}$ and $\mathrm{eV}$, respectively. One can see that the results are very similar to Table 7, which shows that our double-probe measurements are insensitive to the $\Sigma m_{v}$ assumption.

\begin{tabular}{lcccccrc}
\hline & $\Omega_{\mathrm{m}}$ & $H_{0}$ & $\sigma_{8}$ & $\Omega_{\mathrm{k}}$ & $w$ or $w_{0}$ & $w_{\mathrm{a}}$ & $\Sigma m_{\mu}(\mathrm{eV})$ \\
\hline$\Lambda \mathrm{CDM}$ & $0.306 \pm 0.009$ & $68.0 \pm 0.7$ & $0.803 \pm 0.017$ & 0 & -1 & 0 & $<0.12(<0.24)$ \\
$\mathrm{o} \Lambda \mathrm{CDM}$ & $0.307 \pm 0.010$ & $68.2 \pm 0.9$ & $0.796 \pm 0.021$ & $0.003 \pm 0.003$ & -1 & 0 & $<0.19(<0.37)$ \\
$w \mathrm{CDM}$ & $0.295 \pm 0.014$ & $69.5 \pm 1.8$ & $0.798 \pm 0.023$ & 0 & $-1.10 \pm 0.10$ & 0 & $<0.27(<0.53)$ \\
$\mathrm{o} w \mathrm{CDM}$ & $0.296 \pm 0.015$ & $70.1 \pm 2.3$ & $0.781 \pm 0.033$ & $0.003 \pm 0.004$ & $-1.13 \pm 0.14$ & 0 & $<0.45(<0.91)$ \\
$w_{0} w_{\mathrm{a}} \mathrm{CDM}$ & $0.307 \pm 0.020$ & $68.5 \pm 2.3$ & $0.782 \pm 0.028$ & 0 & $-0.92 \pm 0.22$ & $-0.77 \pm 0.73$ & $<0.39(<0.63)$ \\
$\mathrm{o} w_{0} w_{\mathrm{a}} \mathrm{CDM}$ & $0.302 \pm 0.021$ & $69.4 \pm 2.8$ & $0.775 \pm 0.034$ & $0.002 \pm 0.004$ & $-1.01 \pm 0.28$ & $-0.53 \pm 0.88$ & $<0.47(<0.93)$ \\
\hline
\end{tabular}

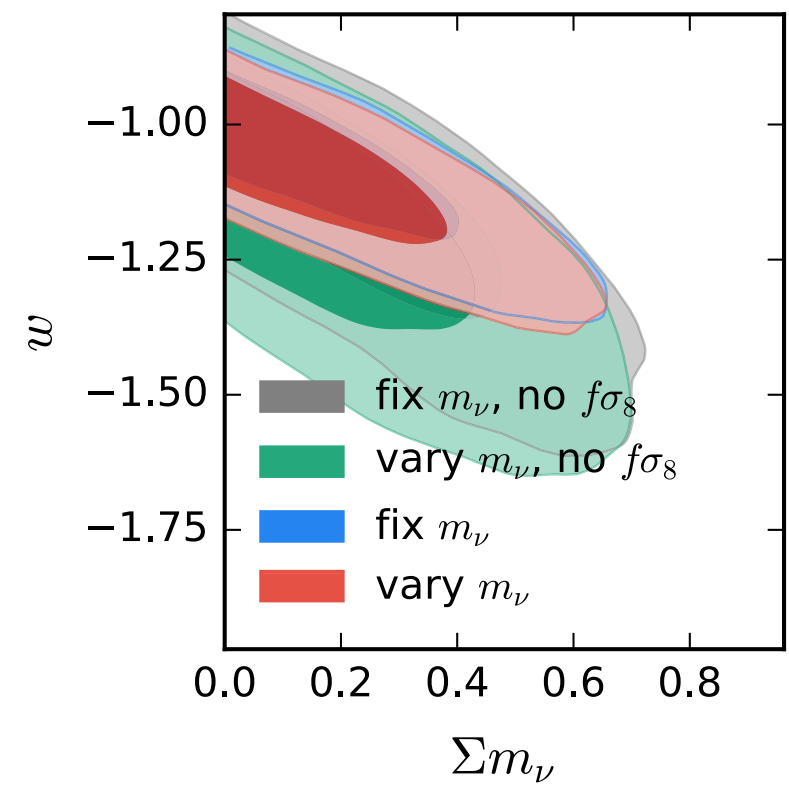

Figure 7. Comparison of 2D contours for 68 and 95 percent confidence level on $\Sigma m_{v}$ and $w$ from the double-probe methodology using covariance matrix from first step varying and fixing neutrinos. One can see that the constraints are insensitive to the assumption of $\Sigma m_{v}$. We also show the results from double-probe measurement excluding $f(z) \sigma_{8}(z)$. It is easy to check that $f(z) \sigma_{8}(z)$ improve the constraint on $w$ but not $\Sigma m_{\nu}$.

and $z_{*}$ is the redshift to the photon-decoupling surface given by CAMB (Lewis et al. 2000).

The angular comoving distance to an object at redshift $z$ is given by

$r(z)=c H_{0}^{-1}\left|\Omega_{\mathrm{k}}\right|^{-1 / 2} F\left[\left|\Omega_{\mathrm{k}}\right|^{1 / 2} \Gamma(z)\right]$,

where

$\Gamma(z)=\int_{0}^{z} \frac{\mathrm{d} z^{\prime}}{E\left(z^{\prime}\right)}$, and $E(z)=H(z) / H_{0}$,

and $F(x)=\sin (x), x, \sinh (x)$ for $\Omega_{\mathrm{k}}<0, \Omega_{\mathrm{k}}=0$ and $\Omega_{\mathrm{k}}>0$ respectively. It has a simple relation with the angular diameter distance $D_{\mathrm{A}}(z)=r(z) /(1+z)$.

In addition to the shift parameters, we include also the scalar index and amplitude of the power-law primordial fluctuation, $n_{\mathrm{S}}$ and $A_{\mathrm{s}}$, to summarize the CMB information.

From the measured parameters $\left\{\Omega_{\mathrm{c}} h^{2}, \Omega_{\mathrm{b}} h^{2}, n_{\mathrm{s}}, \log \left(A_{\mathrm{s}}\right), \theta, \tau\right.$, $\left.\Omega_{\mathrm{k}}, w, H(z), D_{\mathrm{A}}(z), \beta(z), b \sigma_{8}(z), b(z)\right\}$, we derive the parameters $\{R$, $\left.l_{\mathrm{a}}, \Omega_{\mathrm{b}} h^{2}, n_{\mathrm{s}}, \log \left(10^{10} A_{\mathrm{s}}\right), \Omega_{\mathrm{k}}, H(z) r_{\mathrm{s}} / r_{\mathrm{s}, \text { fid }}, D_{\mathrm{A}}(z) r_{\mathrm{s}, \text { fid }} / r_{\mathrm{s}}, f(z) \sigma_{8}(z)\right\}$ to summarize the joint data set of Planck and BOSS galaxy sample.

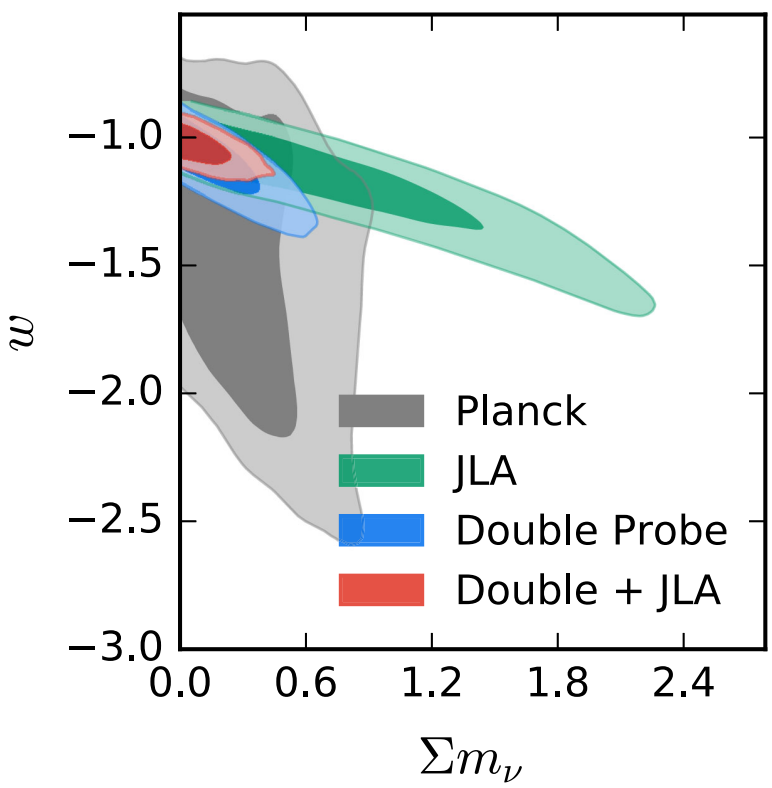

Figure 8. 2D marginalized contours for 68 and 95 per cent confidence level for $w$ and $\Sigma m_{v}$ ( $w \mathrm{CDM}$ model assumed) from Planck-only (grey), double probe (blue), JLA (green) and double probe + JLA (red).

Tables 1 and 2 show the measured values and their normalized covariance. A normalized covariance matrix is defined by

$N_{i j}=\frac{C_{i j}}{\sqrt{C_{i i} C_{j j}}}$,

where $C_{i j}$ is the covariance matrix.

Note here that we have not assumed information from any dark energy model, and that we did not need to add any previous information from $\Omega_{\mathrm{b}} h^{2}, n_{\mathrm{s}}$ or $\Omega_{\mathrm{m}} h^{2}$, thus removing any possible concern over whether these 'informative priors' will have an effect on the measurement. This allows us to obtain any weak degeneracies between the CMB and LSS parameters. The next logical step is then to take these measurements and covariance matrix and use them within the context of a cosmological model to constrain its cosmological parameters. We do this in the next section.

To conveniently compare with other measurements using CMASS sample within $0.43<z<0.7$ (we are using 0.43 $<z<0.75$ ), we extrapolated our measurements at $z=0.57$ : $H(0.57) r_{\mathrm{s}} / r_{\mathrm{s}, \text { fid }}=96.7 \pm 3.1 \mathrm{~km} \mathrm{~s}^{-1} \mathrm{Mpc}^{-1}$ and $D_{\mathrm{A}}(0.57) r_{\mathrm{s}, \text { fid }} / r_{\mathrm{s}}$ $=1405 \pm 25 \mathrm{Mpc}$ (see table 9 of Alam et al. 2016). We checked the impact of redshift evolution on the relevant quantities used in this study $H(z) / H^{\mathrm{fid}}(z), D_{\mathrm{A}}(z) / D_{\mathrm{A}}^{\mathrm{fid}}(z)$ and $f(z) \sigma_{8}(z)$ and found it 
Table 9. Constraints on cosmological parameters from the full-likelihood analysis of the joint data set. $\Sigma m_{\nu}$ is one of the parameters to be constrained. Planck data include lensing with $A_{\mathrm{L}}=1$. The overall shape information of the monopole of the correlation function from the BOSS galaxy clustering is included. We show 68 per cent $1 \mathrm{D}$ marginalized constraints for all the parameters. We provide also 95 per cent constraints for the neutrino masses in the parentheses. The units of $H_{0}$ and $\Sigma m_{v}$ are $\mathrm{km} \mathrm{s}^{-1} \mathrm{Mpc}^{-1}$ and eV, respectively (see Section 7.2 and Fig. 9).

\begin{tabular}{lccccccc}
\hline & $\Omega_{\mathrm{m}}$ & $H_{0}$ & $\sigma_{8}$ & $\Omega_{\mathrm{k}}$ & $w$ or $w_{0}$ & $w_{\mathrm{a}}$ & $\Sigma m_{\mu}(\mathrm{eV})$ \\
\hline$\Lambda \mathrm{CDM}$ & $0.308 \pm 0.011$ & $67.7 \pm 0.9$ & $0.801 \pm 0.017$ & 0 & -1 & 0 & $<0.22(<0.32)$ \\
$\mathrm{o} \Lambda \mathrm{CDM}$ & $0.313 \pm 0.013$ & $67.9 \pm 1.1$ & $0.792 \pm 0.020$ & $0.004 \pm 0.004$ & -1 & 0 & $0.25_{-0.17}^{+0.13}(<0.49)$ \\
$w \mathrm{CDM}$ & $0.293 \pm 0.016$ & $70.1 \pm 2.0$ & $0.808 \pm 0.019$ & 0 & $-1.15 \pm 0.11$ & 0 & $0.30_{-0.14}^{+0.17}(<0.52)$ \\
$\mathrm{o} w \mathrm{CDM}$ & $0.299 \pm 0.019$ & $70.0 \pm 2.4$ & $0.795 \pm 0.021$ & $0.004 \pm 0.004$ & $-1.14 \pm 0.13$ & 0 & $0.40_{-0.17}^{+0.17}\left({ }_{-0.33}^{+0.34}\right)$ \\
$w_{0} w_{\mathrm{a}} \mathrm{CDM}$ & $0.316 \pm 0.023$ & $67.8 \pm 2.5$ & $0.785 \pm 0.023$ & 0 & $-0.87 \pm 0.23$ & $-0.96 \pm 0.68$ & $0.36_{-0.15}^{+0.17}\left({ }_{-0.29}^{+0.26}\right)$ \\
$\mathrm{o} w_{0} w_{\mathrm{a}} \mathrm{CDM}$ & $0.313 \pm 0.026$ & $68.4 \pm 2.8$ & $0.787 \pm 0.027$ & $0.002 \pm 0.004$ & $-0.91 \pm 0.26$ & $-0.82 \pm 0.77$ & $0.39_{-0.15}^{+0.15}\left({ }_{-0.32}^{+0.32}\right)$ \\
\hline
\end{tabular}

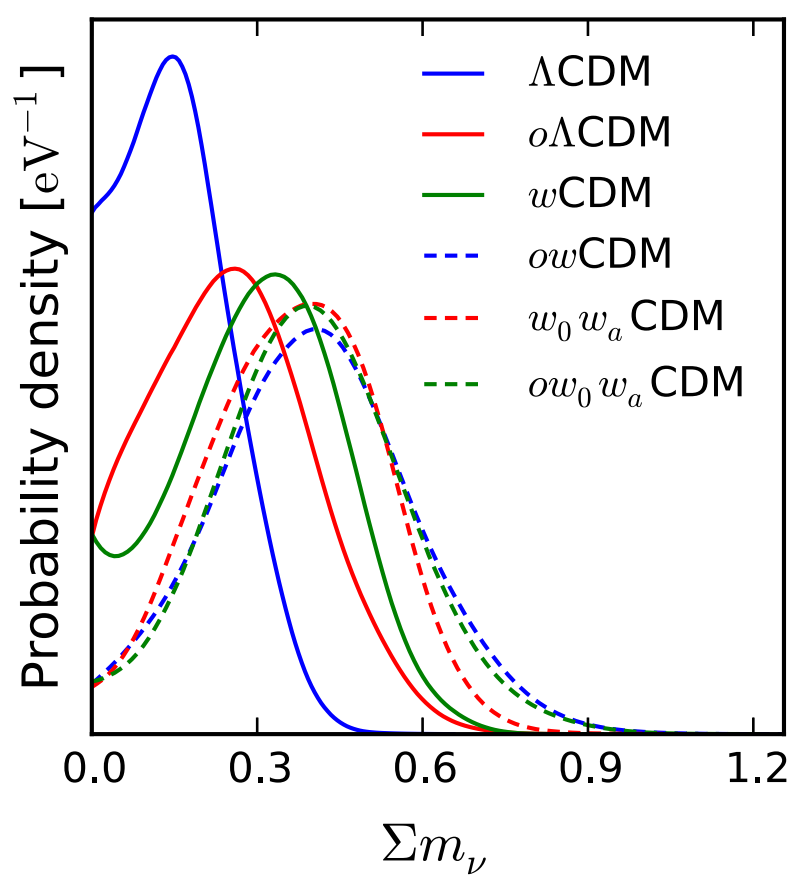

Figure 9. Probability density for $\Sigma m_{v}$ from the full-likelihood analysis of the joint data set. $\Sigma m_{v}$ is one of the parameters to be constrained. Planck data including lensing with $A_{\mathrm{L}}=1$. The overall shape information of the monopole of the correlation function from the BOSS galaxy clustering is included (see Section 7.2 and Table 9)

negligible between $z_{\text {effective }}=0.59$ and 0.57 taking into account the error bars. Thus, the extrapolation is sufficient to compare with other works at these redshift bins.

\section{CONSTRAIN PARAMETERS OF GIVEN DARK ENERGY MODELS WITH DOUBLE-PROBE RESULTS}

In this section, we describe the steps to combine our results with other data sets assuming some dark energy models. For a given model and cosmological parameters, one can compute $\left\{R, l_{\mathrm{a}}, \Omega_{\mathrm{b}} h^{2}\right.$, $n_{\mathrm{s}}, \log \left(10^{10} A_{\mathrm{s}}\right), \Omega_{\mathrm{k}}, H(z) r_{\mathrm{s}} / r_{\mathrm{s}, \text { fid }}, D_{\mathrm{A}}(z) r_{\mathrm{s}, \text { fid }} / r_{\mathrm{s}}$, take the covariance matrices, $M_{i j}$, CMB + galaxy, of these 12 parameters (galaxy sample are divided into two redshift bins) and obtain $\chi_{\mathrm{CMB}+\text { galaxy }}^{2}$ as

$\chi_{\mathrm{CMB}+\text { galaxy }}^{2}=\Delta_{\mathrm{CMB}+\text { galaxy }}^{\mathrm{T}} M_{\mathrm{CMB}+\text { galaxy }}^{-1} \Delta_{\mathrm{CMB}+\text { galaxy }}$,

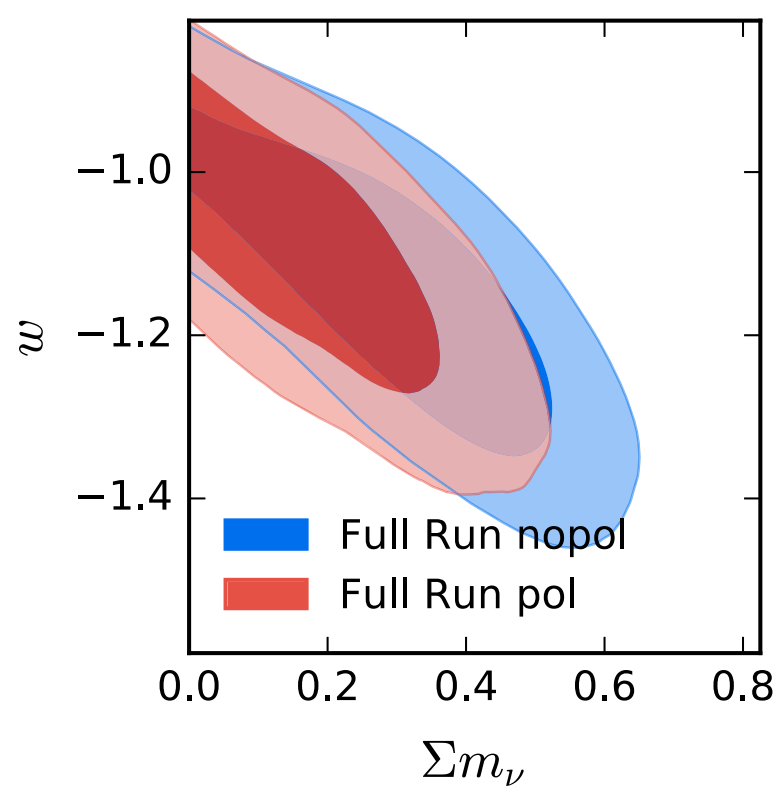

Figure 10. 2D marginalized contours for 68 and 95 per cent confidence level for $w$ and $\Sigma m_{v}$ ( $w$ CDM model assumed) from Planck+BOSS. The blue contours are from full-likelihood analysis without using a polynomial function to remove the overall shape information of monopole; the red contours are from the analysis removing overall shape information with a polynomial function. One can see that the overall shape information shifts the $\Sigma m_{v}$ to a larger value.

where $\mathrm{T}$ indicates the transpose,

$$
\Delta_{\mathrm{CMB}+\text { galaxy }}=\left(\begin{array}{c}
f \sigma_{8}(0.59)-f \sigma_{8}(0.59)_{\mathrm{obs}} \\
H(0.59) r_{\mathrm{s}} / r_{\mathrm{s}, \mathrm{fid}}-H(0.59)_{\mathrm{obs}} r_{\mathrm{s}} / r_{\mathrm{s}, \text { fid }} \\
D_{\mathrm{A}}(0.59) r_{\mathrm{s}, \mathrm{fid}} / r_{\mathrm{s}}-D_{\mathrm{A}}(0.59)_{\mathrm{obs}} r_{\mathrm{s}, \mathrm{fid}} / r_{\mathrm{s}} \\
f \sigma_{8}(0.32)-f \sigma_{8}(0.32)_{\mathrm{obs}} \\
H(0.32) r_{\mathrm{s}} / r_{\mathrm{s}, \mathrm{fid}}-H(0.32)_{\mathrm{obs}} r_{\mathrm{s}} / r_{\mathrm{s}, \text { fid }} \\
D_{\mathrm{A}}(0.32) r_{\mathrm{s}, \mathrm{fid}} / r_{\mathrm{s}}-D_{\mathrm{A}}(0.32)_{\mathrm{obs}} r_{\mathrm{s}, \mathrm{fid}} / r_{\mathrm{s}} \\
R-R_{\mathrm{obs}} \\
l_{\mathrm{a}}-l_{\mathrm{a}, \mathrm{obs}} \\
\Omega_{\mathrm{b}} h^{2}-\Omega_{\mathrm{b}} h_{\mathrm{obs}}^{2} \\
n_{\mathrm{s}}-n_{\mathrm{s}, \mathrm{obs}} \\
\ln \left(10^{10} A_{\mathrm{s}}\right)-\ln \left(10^{10} A_{\mathrm{s}}\right)_{\mathrm{obs}} \\
\Omega_{\mathrm{k}}-\Omega_{\mathrm{k}, \mathrm{obs}}
\end{array}\right.
$$


Table 10. Constraints on cosmological parameters from the full-likelihood analysis of the joint data set. $\Sigma m_{v}$ is one of the parameters to be constrained. Planck data include lensing with $A_{\mathrm{L}}=1$. The overall shape information of the monopole of the correlation function from the BOSS galaxy clustering is removed with a polynomial function. We show 68 per cent $1 \mathrm{D}$ marginalized constraints for all the parameters. We provide also 95 per cent constraints for the neutrino masses in the parentheses. The units of $H_{0}$ and $\Sigma m_{v}$ are $\mathrm{km} \mathrm{s}^{-1} \mathrm{Mpc}^{-1}$ and eV, respectively (see Section 7.2 and Fig. 11).

\begin{tabular}{|c|c|c|c|c|c|c|c|}
\hline & $\Omega_{\mathrm{m}}$ & $H_{0}$ & $\sigma_{8}$ & $\Omega_{\mathrm{k}}$ & $w$ or $w_{0}$ & $w_{\mathrm{a}}$ & $\Sigma m_{\mu}(\mathrm{eV})$ \\
\hline $\mathrm{o} \Lambda \mathrm{CDM}$ & $0.310 \pm 0.012$ & $67.9 \pm 1.0$ & $0.805 \pm 0.017$ & $0.002 \pm 0.003$ & -1 & 0 & $<0.18(<0.36)$ \\
\hline owCDM & $0.300 \pm 0.019$ & $69.1 \pm 2.2$ & $0.813 \pm 0.021$ & $0.001 \pm 0.004$ & $-1.08 \pm 0.12$ & 0 & $<0.21(<0.43)$ \\
\hline$w_{0} w_{\mathrm{a}} \mathrm{CDM}$ & $0.312 \pm 0.027$ & $68.2 \pm 3.1$ & $0.803 \pm 0.028$ & 0 & $-0.91 \pm 0.27$ & $-0.70 \pm 0.79$ & $<0.33(<0.49)$ \\
\hline $\mathrm{o} w_{0} w_{\mathrm{a}} \mathrm{CDM}$ & $0.311 \pm 0.025$ & $68.0 \pm 2.7$ & $0.803 \pm 0.026$ & $0.000 \pm 0.004$ & $-0.92 \pm 0.25$ & $-0.59 \pm 0.78$ & $<0.28(<0.45)$ \\
\hline
\end{tabular}

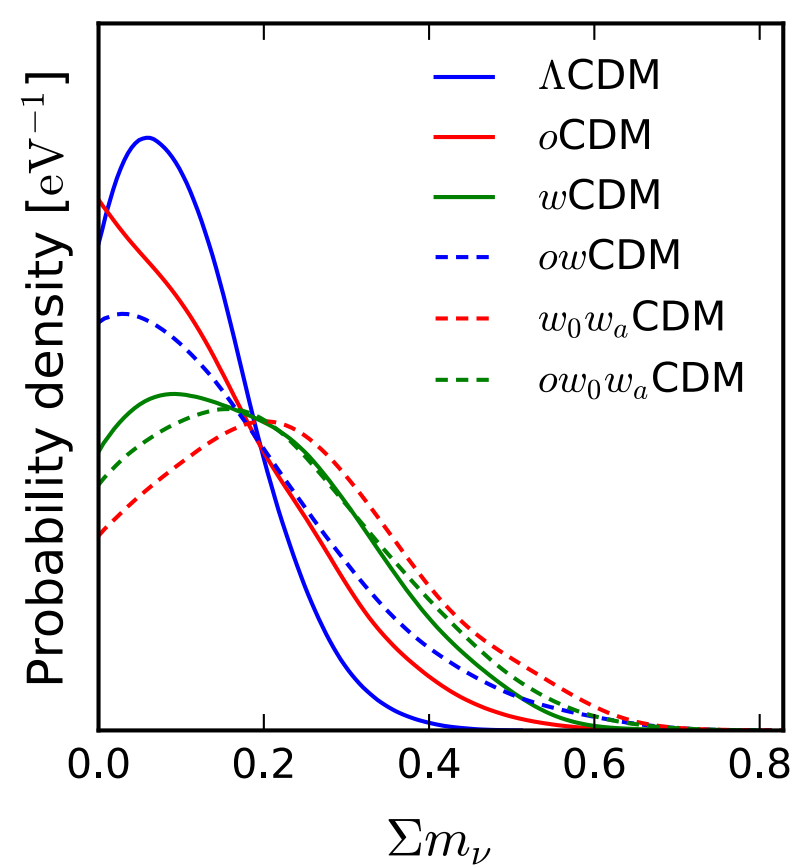

Figure 11. Probability density for $\Sigma m_{v}$ from the full-likelihood analysis of the joint data set. $\Sigma m_{v}$ is one of the parameters to be constrained. Planck data includes lensing with $A_{\mathrm{L}}=1$. The overall shape information of the monopole of the correlation function from the BOSS galaxy clustering is removed with a polynomial function (see Section 7.2 and Table 10).

and the observed parameters are taken from Table 1. The angular diameter distance $D_{\mathrm{A}}(z)$ is given by

$D_{\mathrm{A}}(z)=(1+z) r(z)$,

and the expansion rate of the Universe $H(z)$ is given by

$H(z)$

$=H_{0} \sqrt{\Omega_{\mathrm{m}}(1+z)^{3}+\Omega_{\mathrm{r}}(1+z)^{4}+\Omega_{\mathrm{k}}(1+z)^{2}+\Omega_{\mathrm{X}} X(z)}$,

where $\Omega_{\mathrm{m}}+\Omega_{\mathrm{r}}+\Omega_{\mathrm{k}}+\Omega_{\mathrm{X}}=1$. The dark energy density function $X(z)$ is

$X(z) \equiv \frac{\rho_{\mathrm{X}}(z)}{\rho_{\mathrm{X}}(0)}$.

$f$ is defined in relation to the linear growth factor $D(\tau)$ in the usual way as

$f=\frac{\mathrm{d} \ln D(\tau)}{\mathrm{d} \ln a}=\frac{1}{\mathcal{H}} \frac{\mathrm{d} \ln D(\tau)}{\mathrm{d} \tau}$, where $D$ is the growing solution to the second-order differential equation written in comoving coordinates

$\frac{\mathrm{d}^{2} D(\tau)}{\mathrm{d} \tau^{2}}+\mathcal{H} \frac{\mathrm{d} D(\tau)}{\mathrm{d} \tau}=\frac{3}{2} \Omega_{\mathrm{m}}(\tau) \mathcal{H}^{2}(\tau) D(\tau)$.

We will be writing $\sigma(z, R)$ as

$\sigma^{2}(z, R)=\frac{1}{(2 \pi)^{3}} \int \mathrm{d}^{3} k W^{2}(k R) P(k, z)$

with

$W(k R)=\frac{3}{(k R)^{3}}[\sin (k R)-k R \cos (k R)]$

being the top-hat window function. Thus

$\sigma_{8}(z)=\sigma\left(z, R=8 \mathrm{Mpc} h^{-1}\right)$.

In this way, one just needs to compute linear theory to get $\chi_{\mathrm{CMB}+\text { galaxy }}^{2}$ to reproduce and combine $\mathrm{CMB}$ plus galaxy information, therefore making it a much simpler and faster manner to find constraints. This will have its consequences in the computational time required for doing MCMC.

These equations assume no impact from massive neutrinos, mainly working for the cases of massless or approximately massless neutrinos. When including neutrino species with a given mass, one needs to solve the full Boltzmann hierarchy, as shown in Ma \& Bertschinger (1995) and Lewis \& Challinor (2002).

Table 3 lists the constraints on the parameters of different dark energy models obtained using our double-probe measurements. The results show no tension with the flat $\Lambda \mathrm{CDM}$ cosmological paradigm.

\section{FULL-LIKELIHOOD ANALYSIS FIXING DARK ENERGY MODELS}

To validate our double-probe methodology, we perform the fulllikelihood MCMC analyses fixing dark energy models. The main difference of this approach compared to our double-probe analysis is that it has been given a dark energy model in the first place. In contrast to the double-probe approach, one cannot use the results from the full-likelihood analysis to derive the constraints for the parameters of other dark energy models. Since the dark energy model is fixed, the quantities, $\left\{H(z), D_{\mathrm{A}}(z), \beta(z), b \sigma_{8}(z)\right\}$, would be determined by the input parameters, $\left\{\Omega_{\mathrm{c}} h^{2}, \Omega_{\mathrm{b}} h^{2}, n_{\mathrm{s}}, \log \left(A_{\mathrm{s}}\right), \theta, \tau\right.$, $\left.\Omega_{\mathrm{k}}, w\right\}$, as shown in equations (25), (26), (28) and (32). We show the results in Table 4. In Figs 3-5, we compare these results with our double-probe approach and the single-probe approach (SPM). We find very good agreement among these three approaches. Note that deriving the dark energy model constraints from our doubleprobe measurements is much faster than the full run. For example, using the same machine, it takes $\sim 2.5 \mathrm{~h}$ to obtain the constraints 
Table 11. Constraints on cosmological parameters from the full-likelihood analysis from the joint data set. Both $\Sigma m_{v}$ and $A_{\mathrm{L}}$ are the parameters to be constrained. The overall shape information of the monopole of the correlation function from the BOSS galaxy clustering is removed with a polynomial function. We show 68 per cent 1D marginalized constraints for all the parameters. We provide also 95 per cent constraints for the neutrino masses in the parentheses. The units of $H_{0}$ and $\Sigma m_{v}$ are $\mathrm{km} \mathrm{s}^{-1} \mathrm{Mpc}^{-1}$ and eV, respectively (see Section 7.2 and Fig. 12).

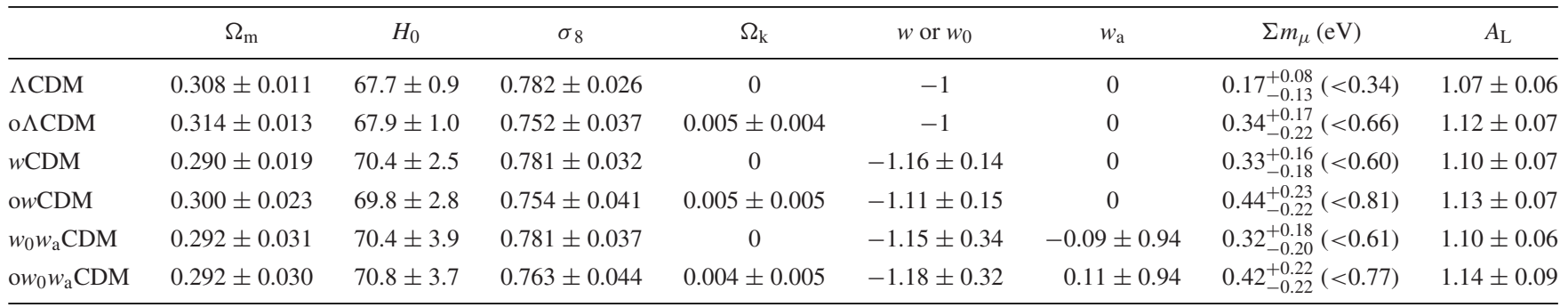

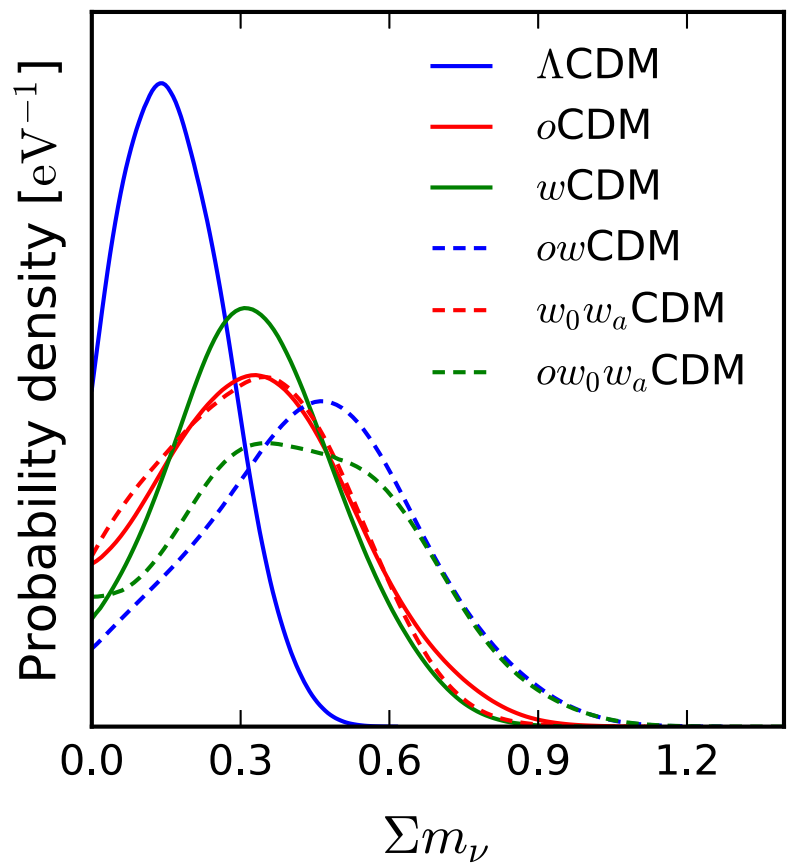

Figure 12. Probability density for $\Sigma m_{v}$ from full-likelihood analysis from the joint data set. Both $\Sigma m_{v}$ and $A_{\mathrm{L}}$ are the parameters to be constrained. The overall shape information of the monopole of the correlation function from the BOSS galaxy clustering is removed with a polynomial function (see Section 7.2 and Table 11). One can see that the maximum of $\Sigma m_{v}$ increases comparing to the cases with fixing $A_{\mathrm{L}}=1$ (see Fig. 11).

for $\Lambda \mathrm{CDM}$ using double-probe measurements, whereas it would take $6 \mathrm{~d}$ to reach similar convergence for the full-likelihood MCMC analysis (slower by a factor of 60). Both cases were done with 16 CPUs for each dark energy model.

Up to this point, we have introduced two methodologies, the double-probe method and a full-likelihood analysis, for extracting cosmological information. Moreover, we are comparing these results with a third methodology already introduced in SPM called single-probe analysis combined with the CMB. We show here motivations for the use of each of them.

(i) Double probe. Joint fit to the LSS data and the CMB constraining the full set of cosmological parameters without the need for extra knowledge of the priors. This methodology allows us to test the prior information content assumed by other probes and gives us the tool to have dark energy-independent measurements from LSS and $\mathrm{CMB}$ combined.

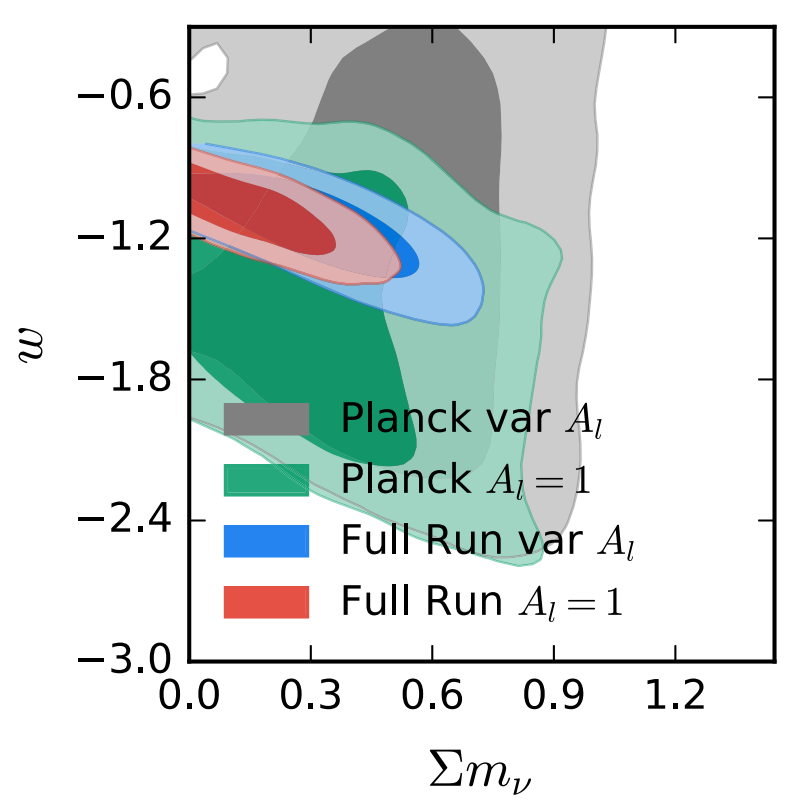

Figure 13. 2D marginalized contours for 68 and 95 percent confidence level for $w$ and $\Sigma m_{v}$ ( $w$ CDM model assumed) from full run methodology and Planck-only for different lensing information used. Grey contours and green contours are from Planck-only with varying $A_{\mathrm{L}}$ and fixing $A_{\mathrm{L}}=1$, respectively; the blue contours and the red contours are from Planck+BOSS with varying $A_{\mathrm{L}}$ and fixing $A_{\mathrm{L}}=1$ respectively using full-likelihood analysis. One can see that $\Sigma m_{v}$ shift to a large value when varying $A_{\mathrm{L}}$ for both data combinations.

(ii) Full (or exact) fit. Fit of cosmological parameter set to LSS and $\mathrm{CMB}$ data, requiring the assumption of a dark energy model (i.e. not going through $D_{\mathrm{A}}, H$ and $f \sigma_{8}$ as intermediate parameters) from the beginning. This methodology provides a tool to check the information content of the data, and we take it to be the true answer to recover from other methodologies as it does not have extra assumptions apart from the dark energy model.

(iii) Single probe $+C M B$ (SPM). Likelihoods are determined from the BOSS measurements of $\left\{D_{\mathrm{A}} r_{\mathrm{s}}^{\mathrm{fid}} / r_{\mathrm{s}}, H r_{\mathrm{s}} / r_{\mathrm{s}}^{\mathrm{fid}}, f \sigma_{8}, \Omega_{\mathrm{m}} h^{2}\right\}$, together with Planck data. This methodology provides, in its first step, measurements from LSS independent of CMB data, thus revealing itself to be a good tool to test possible tensions between data sets. In order to get this independent analysis, wide priors for LSS poorly constrained parameters are assumed.

\section{MEASUREMENTS OF NEUTRINO MASS}

In this section, we will focus on measuring the sum of the neutrino mass $\Sigma m_{v}$ using the different methodologies described in previous 
Table 12. The cosmological constraints including total mass of neutrinos from the single-probe measurements provided by SPM combining with Planck data assuming different dark energy models. We show 68 per cent 1D marginalized constraints for all the parameters. We provide also 95 per cent constraints for the neutrino masses in the parentheses. The units of $H_{0}$ and $\Sigma m_{v}$ are $\mathrm{km} \mathrm{s}^{-1} \mathrm{Mpc}^{-1}$ and eV, respectively (see Section 7.2 and Fig. 14).

\begin{tabular}{|c|c|c|c|c|c|c|c|}
\hline & $\Omega_{\mathrm{m}}$ & $H_{0}$ & $\sigma_{8}$ & $\Omega_{\mathrm{k}}$ & $w$ or $w_{0}$ & $w_{\mathrm{a}}$ & $\Sigma m_{v}$ \\
\hline Planck+BOSS (o $\Lambda \mathrm{CDM})$ & $0.313 \pm 0.011$ & $67.6 \pm 0.9$ & $0.804 \pm 0.016$ & $0.002 \pm 0.004$ & -1 & 0 & $<0.20$ \\
\hline Planck+BOSS (owCDM) & $0.306 \pm 0.014$ & $68.5 \pm 1.6$ & $0.809 \pm 0.018$ & $0.001 \pm 0.004$ & $-1.06 \pm 0.09$ & 0 & $<0.24$ \\
\hline Planck+BOSS $\left(w_{0} w_{\mathrm{a}} \mathrm{CDM}\right)$ & $0.314 \pm 0.021$ & $67.8 \pm 2.2$ & $0.800 \pm 0.022$ & 0 & $-0.91 \pm 0.22$ & $-0.70 \pm 0.75$ & $0.26_{-0.18}^{+0.13}$ \\
\hline Planck+BOSS $\left(\mathrm{o} w_{0} w_{\mathrm{a}} \mathrm{CDM}\right)$ & $0.315 \pm 0.020$ & $67.7 \pm 2.1$ & $0.799 \pm 0.022$ & $-0.001 \pm 0.004$ & $-0.90 \pm 0.21$ & $-0.73 \pm 0.73$ & $0.25_{-0.22}^{+0.08}$ \\
\hline
\end{tabular}

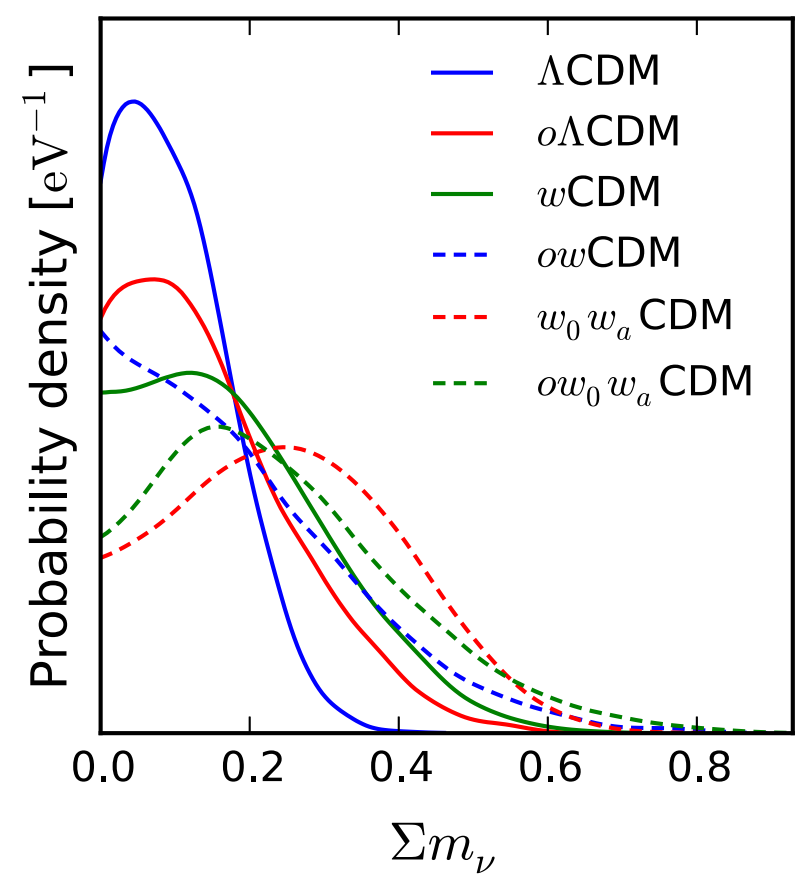

Figure 14. Probability density for $\Sigma m_{v}$ from the single-probe measurements provided by SPM combining with Planck data (with fixing $A_{\mathrm{L}}=1$ ). All the measurements are consistent with $\Sigma m_{v}=0$ (see Section 7.2 and Table 12).

sections. First, we repeat the double-probe analysis from Section 3.3 with an additional free parameter, $\Sigma m_{v}$, and present the constraints on cosmological parameters. Secondly, we repeat the MCMC analysis with the full likelihood of joint data set explained in Section 6. We show that the full shape measurement of the monopole of the galaxy two-point correlation function introduces some detection of neutrino mass inconsistent with other independent experiments. However, since the monopole measurement is sensitive to the observational systematics, we provide with another set of cosmological constraints by removing the full shape information, therefore, showing that there is a degeneracy between neutrino mass measurement and observational systematics modelled as nuisance parameters. Thirdly, we obtain the constraint on $\Sigma m_{v}$ using the single-probe measurement provided by SPM.

\subsection{Measuring neutrino mass using double probe}

Note first that for the study of $m_{v}$, we replace $R=\sqrt{\Omega_{\mathrm{m}} H_{0}^{2}} r\left(z_{*}\right)$ with $\Omega_{\mathrm{bc}} h^{2}=\Omega_{\mathrm{b}} h^{2}+\Omega_{\mathrm{c}} h^{2}$ (e.g. see Aubourg et al. 2015), since $R$ depends directly on $\Omega_{v}$. Thus, we use the following set of parameters from the double-probe analysis while measuring neutrino mass, $\left\{\Omega_{\mathrm{bc}} h^{2}, l_{\mathrm{a}}, \Omega_{\mathrm{b}} h^{2}, n_{\mathrm{s}}, \log \left(A_{\mathrm{s}}\right), \Omega_{\mathrm{k}}, H(z), D_{\mathrm{A}}(z), f(z) \sigma_{8}(z)\right\}$.

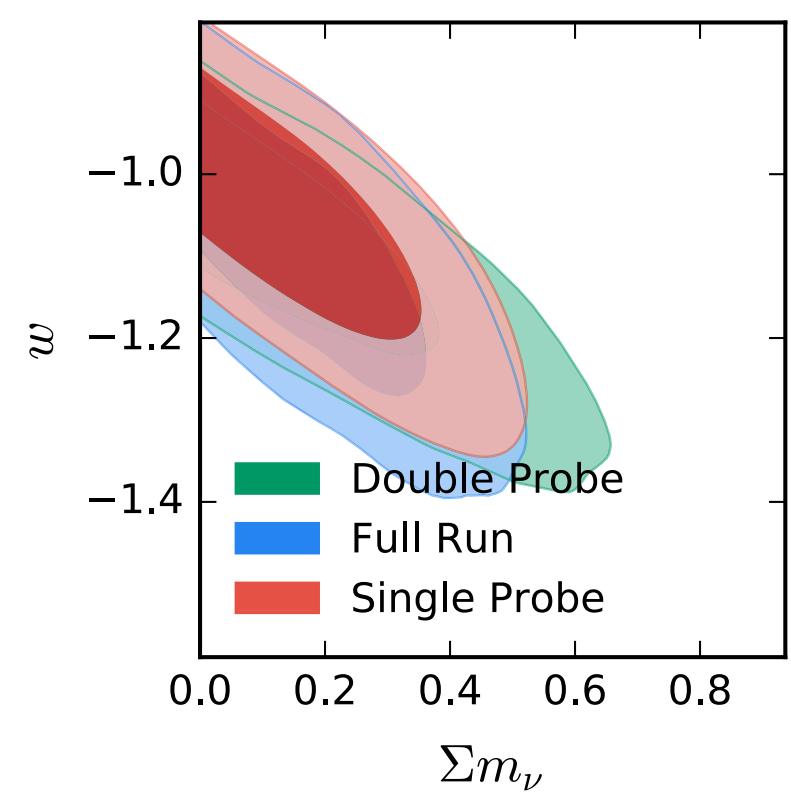

Figure 15. Comparison of 2D contours for 68 and 95 per cent confidence level on $\Sigma m_{v}$ and $w$ from the double-probe, single-probe and full-likelihood analysis approaches. One can see that the constraint on $\Sigma m_{v}$ from the doubleprobe approach is weaker, which is expected. The difference comes from the fact that we do not include $\Sigma m_{v}$ into our summarized set of parameters, so information from Planck is lost.

We repeat the analysis described in Section 3.3, but here we set $\Sigma m_{v}$ to be free instead of setting it to $0.06 \mathrm{eV}$. The results are shown in Tables 5 and 6.

As described in Section 5, one can constrain the parameters of given dark energy models using Tables 5 and 6. Table 7 presents the cosmological parameter constraints assuming some simple dark energy models. Fig. 6 displays the probability density for $\Sigma m_{v}$ for different dark energy models. Our measurements of $\Sigma m_{v}$ using double-probe approach are consistent with zero. The upper limit (68 per cent confidence level) varies from 0.1 to $0.35 \mathrm{eV}$ depending on dark energy model.

In addition, we also derive the cosmological constraints by using the results with fixed $\Sigma m_{v}$, i.e. Tables 1 and 2 with $R$ replaced by $\Omega_{\mathrm{bc}} h^{2}$. Different from Table 3 (see Section 5), we include $\Sigma m_{v}$ as one of the parameters to be constrained. The results are shown in Table 8. We find that the results are very similar to Table 7, which shows that our double-probe measurements are insensitive to the $\Sigma m_{v}$ assumption. Fig. 7 presents this point in a clear way by comparing the $2 \mathrm{D}$ contours when including a covariance matrix varying $\Sigma m_{v}$ (using Tables 5 and 6) or fixing $\Sigma m_{v}$ (using Tables 1 and 2). We see that they lie on top of each other. Moreover, Fig. 7 also exhibits the constraint given by $f \sigma_{8}$ on the $\Sigma m_{v}$ and $w$ parameters. 
Table 13. Constraints on cosmological parameters from the full-likelihood analysis of the joint (Planck and BOSS DR12) and JLA data sets assuming variable $\Sigma m_{v}$. Planck data include lensing with $A_{\mathrm{L}}=1$. The overall shape information of the monopole of the correlation function from the BOSS galaxy clustering is removed with a polynomial function. We show 68 per cent $1 \mathrm{D}$ marginalized constraints for all the parameters. We provide also 95 per cent constraints for the neutrino masses in the parentheses. The units of $H_{0}$ and $\Sigma m_{\nu}$ are $\mathrm{km} \mathrm{s}^{-1} \mathrm{Mpc}^{-1}$ and eV, respectively (see Section 7.2 and Fig. 16).

\begin{tabular}{|c|c|c|c|c|c|c|c|}
\hline & $\Omega_{\mathrm{m}}$ & $H_{0}$ & $\sigma_{8}$ & $\Omega_{\mathrm{k}}$ & $w$ or $w_{0}$ & $w_{\mathrm{a}}$ & $\Sigma m_{\mu}(\mathrm{eV})$ \\
\hline $\mathrm{o} \Lambda \mathrm{CDM}$ & $0.309 \pm 0.010$ & $67.9 \pm 0.9$ & $0.807 \pm 0.016$ & $0.001 \pm 0.004$ & -1 & 0 & $<0.17(<0.33)$ \\
\hline owCDM & $0.307 \pm 0.013$ & $68.3 \pm 1.4$ & $0.808 \pm 0.019$ & $0.001 \pm 0.004$ & $-1.03 \pm 0.06$ & 0 & $<0.20(<0.43)$ \\
\hline$w_{0} w_{\mathrm{a}} \mathrm{CDM}$ & $0.309 \pm 0.014$ & $68.2 \pm 1.3$ & $0.807 \pm 0.019$ & 0 & $-0.92 \pm 0.12$ & $-0.64 \pm 0.56$ & $<0.26(<0.43)$ \\
\hline $\mathrm{o} w_{0} w_{\mathrm{a}} \mathrm{CDM}$ & $0.310 \pm 0.013$ & $68.0 \pm 1.3$ & $0.803 \pm 0.019$ & $0.000 \pm 0.004$ & $-0.91 \pm 0.11$ & $-0.63 \pm 0.59$ & $<0.27(<0.46)$ \\
\hline
\end{tabular}

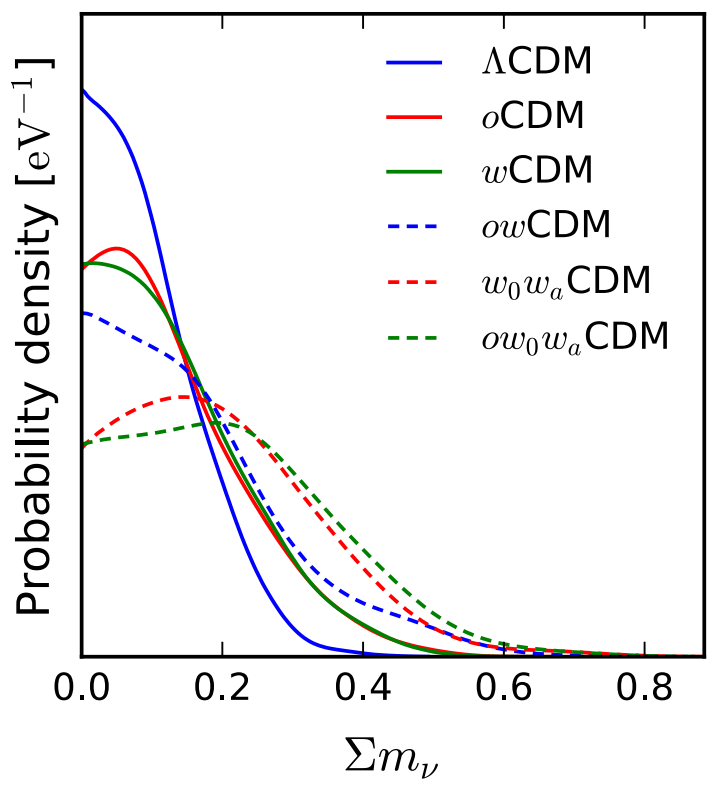

Figure 16. Probability density for $\Sigma m_{v}$ from the full-likelihood analysis measurement for joint and JLA data sets. We assume lensing likelihood with fixed $A_{\mathrm{L}}=1$. All the measurements are consistent with $\Sigma m_{v}=0$ (see Section 7.2 and Table 13).

We find that the constraint on $w$ becomes tighter while that in $\Sigma m_{v}$ stays the same when including the $f \sigma_{8}$ information. This is a good news for future experiments as their power on the neutrino constraint would not highly rely on the growth rate measurements that are more sensitive to the observational systematics.

Furthermore, we have also checked the impact of adding Type Ia supernovae (SNIa) data, dubbed joint light-curve analysis (JLA; Betoule et al. 2014) and find that the upper limit of $\Sigma m_{v}$ decreases because SNIa breaks the degeneracy of the constraint from Planck+BOSS (see Fig. 8). In this way, we can get tighter constraints on the upper limit by including SNIa data.

\subsection{Measuring neutrino mass using full-likelihood analysis}

We perform the same full MCMC analysis using the joint full likelihood of Planck and BOSS data as described in Section 6 to obtain the cosmological parameter constraints including $\Sigma m_{v}$. Table 9 presents the results. We show the probability density for $\Sigma m_{v}$ in Fig. 9 first without accounting for the potential systematics coming mainly from stellar density, seeing and extinction (Ross et al. 2017). We find more than $2 \sigma$ detection of non-zero $\Sigma m_{v}$ assuming models without fixing $w$ to be -1 , which is in clear contradiction with previous measurements from BAO. However, we find that the detection actually comes from the monopole shape of galaxy correlation function that is sensitive to some observational systematics (e.g. see Ross et al. 2012; Chuang et al. 2016b). Fig. 10 shows that the $\Sigma m_{v}$ detection decreases when adding a polynomial to remove the full shape information of monopole (see SPM) and is in agreement with previous works. Henceforth, when constraining $\Sigma m_{v}$, we take care of accounting for unknown systematics as they will affect the measurement. We propose to use this conservative approach of marginalizing over unknown systematics as the reliable results. We run again the full MCMC analysis to obtain the constraint on $\Sigma m_{v}$ without including the full shape information. The results are presented in Table 10. The probability density for $\Sigma m_{v}$ is shown in Fig. 11. One can see again that the detections of $\Sigma m_{v}$ decrease. In addition, the upper limits in Fig. 11 are lower than Fig. 6, which is expected. Since we do not include the parameter $\Sigma m_{v}$ when summarizing the information of double probe, the $\Sigma m_{v}$ constraint from Planck is lost.

Table 11 and Fig. 12 display the constraints measured when allowing the CMB lensing amplitude parameter $A_{\mathrm{L}}$ to vary. Fig. 13 shows that Planck data shift $\Sigma m_{v}$ measurement to higher values providing a higher detection from the combined data analysis when letting $A_{\mathrm{L}}$ free. Thus, we find again $\sim 2 \sigma$ detection even without accounting for the full shape of the monopole from the correlation function. Note that this shift is not due to observational systematics any more, it is coming from CMB only.

\subsection{Measuring neutrino mass using measurements from single-probe analysis (SPM)}

We use the single-probe measurement provided by SPM combining with Planck (fixing $A_{\mathrm{L}}=1$ ) and obtain the constraint of $\Sigma m_{v}$. Table 12 shows the cosmological parameter constraints including $\Sigma m_{v}$ for different dark energy models. The probability densities for $\Sigma m_{v}$ are shown in Fig. 14. One can see that they are consistent with Fig. 11. We have checked that there would be some detection of neutrino mass while allowing $A_{\mathrm{L}}$ to be free as seen in the case of full-likelihood analysis (see Section 7.2).

Fig. 15 presents the comparison between the three different methodologies. The three approaches agree very well with some subtle differences. One can see that the constraint on $\Sigma m_{v}$ from the double-probe approach is weaker. The difference comes from the fact that we do not include $\Sigma m_{v}$ into our summarized set of parameters, so that information from Planck is lost. On the other hand, both single-probe and full-likelihood analysis include full Planck information and their measurements are very similar.

\subsection{Combination with SNIa data}

We combine our measurements using the full-likelihood approach with those from SNIa data, JLA (Betoule et al. 2014) that combined SNe from the SDSS-II Supernova Survey (Sako et al. 2014) and the Supernova Legacy Survey 3-year data set (Conley et al. 2011) 
Table 14. Constraints on cosmological parameters from the full-likelihood analysis of the joint (Planck and BOSS DR12) and JLA data sets assuming variable $\Sigma m_{v}$. Planck data include lensing varying $A_{\mathrm{L}}$. The overall shape information of the monopole of the correlation function from the BOSS galaxy clustering is removed with a polynomial function. We show 68 per cent 1D marginalized constraints for all the parameters. We provide also 95 per cent constraints for the neutrino masses in the parentheses. The units of $H_{0}$ and $\Sigma m_{v}$ are $\mathrm{km} \mathrm{s}^{-1} \mathrm{Mpc}^{-1}$ and eV, respectively (see Section 7.2 and Fig. 17).

\begin{tabular}{|c|c|c|c|c|c|c|c|c|}
\hline & $\Omega_{\mathrm{m}}$ & $H_{0}$ & $\sigma_{8}$ & $\Omega_{\mathrm{k}}$ & $w$ or $w_{0}$ & $w_{\mathrm{a}}$ & $\Sigma m_{\mu}(\mathrm{eV})$ & $A_{L}$ \\
\hline $\mathrm{o} \Lambda \mathrm{CDM}$ & $0.311 \pm 0.013$ & $68.0 \pm 1.1$ & $0.755 \pm 0.037$ & $0.005 \pm 0.005$ & -1 & 0 & $0.32_{-0.23}^{+0.16}(<0.63)$ & $1.12 \pm 0.08$ \\
\hline owCDM & $\begin{array}{l}0.310 \pm 0.012 \\
0310+0.013\end{array}$ & $\begin{array}{l}68.5 \pm 1.3 \\
681+12\end{array}$ & $\begin{array}{l}0.148 \pm 0.038 \\
0.769+0.035\end{array}$ & $\begin{array}{r}0.000 \pm \\
0\end{array}$ & $\begin{array}{l}-1.04 \pm 0.00 \\
-0.93+0.12\end{array}$ & $-070+061$ & $\begin{array}{l}0.40_{-0.25}(<0.10) \\
033+0.16(<0.61)\end{array}$ & $\begin{array}{l}1.13 \pm 0.08 \\
1.09+0.07\end{array}$ \\
\hline $\begin{array}{l}w_{0} w_{\mathrm{a}} \mathrm{CDM} \\
\mathrm{o} w_{0} w_{3} \mathrm{CDM}\end{array}$ & $\begin{array}{l}0.310 \pm 0.013 \\
0.310 \pm 0.016\end{array}$ & $68.5 \pm 1.6$ & $\begin{array}{l}0.109 \pm 0.033 \\
0.756 \pm 0.037\end{array}$ & $0.004+0.005$ & $\begin{array}{l}-0.93 \pm 0.12 \\
-0.97 \pm 0.14\end{array}$ & $\begin{array}{l}-0.10 \pm 0.01 \\
-0.41 \pm 0.67\end{array}$ & $0.33^{+0.20}(<0.74)$ & $\begin{array}{l}1.09 \pm 0.01 \\
1.12 \pm 0.08\end{array}$ \\
\hline
\end{tabular}

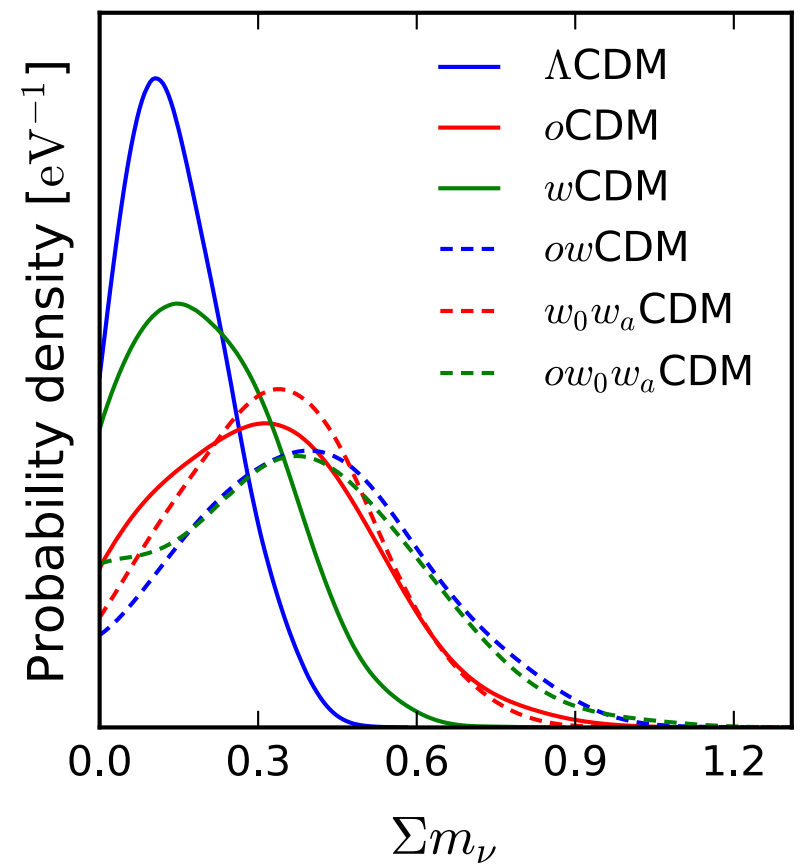

Figure 17. Probability density for $\Sigma m_{v}$ from the full-likelihood analysis measurement for joint and JLA data sets. We assume lensing likelihood with variable $A_{\mathrm{L}}$ (see Section 7.2 and Table 14).

together with local and high- $z$ data sets. The combination of BAO and $\mathrm{SN}$ measurements is important for constraining the low-redshift distance scale where BAO has difficulties due to the small-volume sample. As seen in Fig. 8, SN data break some degeneracies providing tighter constraints on $\Sigma m_{v}$. Results can be found in Table 13 and Fig. 16 for the case of fixing $A_{\mathrm{L}}=1$ and Table 14 and Fig. 17 for the case of varying $A_{\mathrm{L}}$. When adding SNIa data, we get tighter upper limits, e.g. $\Sigma m_{v}<0.12$ against $\Sigma m_{v}<0.14$ in $\Lambda$ CDM with $A_{\mathrm{L}}=1$. We point out that the constraints we obtained are still not sufficient to distinguish between normal and inverted hierarchy.

\section{SUMMARY}

In this work, we have studied and compared three different ways of extracting cosmological information from the combined data sets of Planck 2015 and the BOSS DR12, taking care to avoid imposing informative priors on cosmological parameters when combining these data.

First, we have extracted the dark energy model-independent cosmological constraints from the joint data sets of BOSS galaxy sample and Planck CMB measurement. We measure the mean values and covariance matrix of $\left\{R, l_{\mathrm{a}}, \Omega_{\mathrm{b}} h^{2}, n_{\mathrm{s}}, \log \left(A_{\mathrm{s}}\right), \Omega_{\mathrm{k}}, H(z), D_{\mathrm{A}}(z)\right.$, $\left.f(z) \sigma_{8}(z)\right\}$, which give an efficient summary of Planck data and twopoint statistics from BOSS galaxy sample (see Table 1). We called this methodology the 'double-probe' approach since it combines two data sets to minimize the informative priors needed for the cosmological parameters. We found that double-probe measurements are insensitive to the assumption of neutrino mass (fixed or not). However, the parameter $R$ should be replaced by $\Omega_{\mathrm{bc}} h^{2}$ while setting $\Sigma m_{v}$ to be free.

Secondly, we performed the full-likelihood analysis from the joint data set of Planck and BOSS assuming some simple dark energy models. By comparing these results with those from the doubleprobe approach, we have demonstrated that the double-probe approach provides robust cosmological parameter constraints that can be conveniently used to study dark energy models. Using our results, we obtain $\Omega_{\mathrm{m}}=0.304 \pm 0.009, H_{0}=68.2 \pm 0.7$ and $\sigma_{8}=0.806$ \pm 0.014 assuming $\Lambda \mathrm{CDM}, \Omega_{\mathrm{k}}=0.002 \pm 0.003$ assuming $\mathrm{oCDM}$, $w=-1.04 \pm 0.06$ assuming $w \mathrm{CDM}, \Omega_{\mathrm{k}}=0.002 \pm 0.003$ and $w=-1.00 \pm 0.07$ assuming $\mathrm{o} w \mathrm{CDM}$, and $w_{0}=-0.84 \pm 0.22$ and $w_{\mathrm{a}}=-0.66 \pm 0.68$ assuming $w_{0} w_{\mathrm{a}} \mathrm{CDM}$. The results show no tension with the flat $\Lambda \mathrm{CDM}$ cosmological paradigm. Note that deriving the dark energy model constraints from our double-probe measurements is much faster than the full run. For example, it takes $\sim 2.5 \mathrm{~h}$ to obtain the constraints for $\Lambda \mathrm{CDM}$ using double-probe measurements, but it takes $6 \mathrm{~d}$ to reach a similar convergence for the full MCMC run (slower by a factor of 60) using 16 CPUs in each case.

We have extended our study to measure the sum of neutrino mass using these different methodologies including double-probe analysis (introduced in this study), full-likelihood analysis and singleprobe analysis. We found that the double probe constrains the neutrino mass more weakly, since it does not include the measuring power on the neutrino mass from Planck data. While including lensing information, we have performed the analyses with varying $A_{\mathrm{L}}$ or fixed $A_{\mathrm{L}}=1$. We found that varying $A_{\mathrm{L}}$ shifts $\Sigma m_{v}$ to larger values. From the full-likelihood analysis with varying $A_{\mathrm{L}}$, we obtained $\Sigma m_{v}=0.17_{-0.13}^{+0.08}$ assuming $\Lambda \mathrm{CDM}, \Sigma m_{v}=0.34_{-0.22}^{+0.17}$ assuming $\mathrm{o} \Lambda \mathrm{CDM}, \Sigma m_{v}=0.33_{-0.18}^{+0.16}$ assuming $w \mathrm{CDM}$ and $\Sigma m_{v}=0.44_{-0.22}^{+0.23}$ assuming owCDM. We found $\sim 2 \sigma$ detection of $\Sigma m_{v}$ when allowing $w$ and $\Omega_{k}$ to be free.

In addition, when performing the full-likelihood analysis, we found that the overall shape of correlation function contributed to the detection of neutrino mass significantly. However, since we do not have high confidence on the overall shape because of the potential observational systematics, we removed the overall shape information to be conservative. The numbers provided above have been obtained without the overall shape information. Our study has shown that one should be cautious regarding the impact of 
observational systematics when constraining the neutrino mass using the LSS measurements.

\section{ACKNOWLEDGEMENTS}

MPI would like to thank Denis Tramonte and Rafael Rebolo for useful discussions. MPI and CC thank David Hogg, Savvas Nesseris and Yun Wang for useful discussions. CC and FP acknowledge support from the Spanish MICINNs Consolider-Ingenio 2010 Programme under grant MultiDark CSD2009-00064 and grant AYA2010-21231-C02-01. CC was also supported by the Comunidad de Madrid under grant HEPHACOS S2009/ESP-1473. CC was supported as a MultiDark fellow. MPI acknowledges support from MINECO under the grant AYA2012-39702-C02-01. GR is supported by the National Research Foundation of Korea (NRF) through NRF-SGER 2014055950 funded by the Korean Ministry of Education, Science and Technology (MoEST), and by the faculty research fund of Sejong University in 2016. MVM acknowledges support from Programa de Apoyo a Proyectos de Investigación e Innovación Tecnológica (PAPITT) No IA102516, from Proyecto Conacyt Fronteras No 281 and from Proyecto LANCAD-UNAMDGTIC-319.

We acknowledge the use of the CURIE supercomputer at Très Grand Centre de Calcul du CEA in France through the French participation into the PRACE research infrastructure, the SuperMUC supercomputer at Leibniz Supercomputing Centre of the Bavarian Academy of Science in Germany, the TEIDE High Performance Computing facilities in Spain and the Hydra cluster at Instituto de Física Teórica (UAM/CSIC) in Spain. TeideHPC facilities are provided by the Instituto Tecnológico y de Energías Renovables (ITER, SA).

Funding for SDSS-III has been provided by the Alfred P. Sloan Foundation, the Participating Institutions, the National Science Foundation and the US Department of Energy Office of Science. The SDSS-III website is http://www.sdss3.org/.

SDSS-III is managed by the Astrophysical Research Consortium for the Participating Institutions of the SDSS-III Collaboration including the University of Arizona, the Brazilian Participation Group, Brookhaven National Laboratory, Carnegie Mellon University, University of Florida, the French Participation Group, the German Participation Group, Harvard University, the Instituto de Astrofisica de Canarias, the Michigan State/Notre Dame/JINA Participation Group, Johns Hopkins University, Lawrence Berkeley National Laboratory, Max Planck Institute for Astrophysics, Max Planck Institute for Extraterrestrial Physics, New Mexico State University, New York University, Ohio State University, Pennsylvania State University, University of Portsmouth, Princeton University, the Spanish Participation Group, University of Tokyo, University of Utah, Vanderbilt University, University of Virginia, University of Washington and Yale University.

\section{REFERENCES}

Abazajian K. et al., 2005, AJ, 129, 1755

Abazajian K. N. et al., 2009, ApJS, 182, 543

Ade P. A. R. et al., 2014a, A\&A, 571, A1

Ade P. A. R. et al., 2014b, A\&A, 571, A20

Aihara H. et al., 2011, ApJS, 193, 29

Alam S. et al., 2015a, ApJS, 219, 12

Alam S., Ho S., Vargas-Magaña M., Schneider D. P., 2015b, MNRAS, 453, 1754

Alam S. et al., 2016, MNRAS, preprint (arXiv:1607.03155)
Alcock C., Paczynski B., 1979, Nature, 281, 358

Anderson L. et al., 2014, MNRAS, 441, 24

Aubourg É. et al., 2015, Phys. Rev. D, 92, 123516

Battye R. A., Moss A., 2014, Phys. Rev. Lett., 112, 051303

Bennett C. L. et al., 2013, ApJS, 208, 20

Bernardeau F., 1994, ApJ, 427, 51

Bersanelli M. et al., 2010, A\&A, 520, A4

Betoule M. et al., 2014, A\&A, 568, A22

Beutler F. et al., 2014a, MNRAS, 443, 1065

Beutler F. et al., 2014b, MNRAS, 444, 3501

Beutler F. et al., 2017a, MNRAS, 464, 3409

Beutler F. et al., 2017b, MNRAS, 466, 2242

Bolton A. S. et al., 2012, AJ, 144, 144

Bouchet F. R., Colombi S., Hivon E., Juszkiewicz R., 1995, A\&A, 296, 575

Buchert T., 1994, MNRAS, 267, 811

Burenin R. A., 2013, Astron. Lett., 39, 357

Catelan P., 1995, MNRAS, 276, 115

Chuang C.-H., Wang Y., 2012, MNRAS, 426, 226

Chuang C.-H., Wang Y., 2013a, MNRAS, 431, 2634

Chuang C.-H., Wang Y., 2013b, MNRAS, 435, 255

Chuang C.-H., Wang Y., Hemantha M. D. P., 2012, MNRAS, 423, 1474

Chuang C.-H. et al., 2013, MNRAS, 433, 3559

Chuang C.-H. et al., 2016a, preprint (arXiv:1607.03151)

Chuang C.-H. et al., 2016b, MNRAS, 461, 378

Colless M. et al., 2001, MNRAS, 328, 1039

Colless M. et al., 2003, preprint (astro-ph/0306581)

Conley A. et al., 2011, ApJS, 192, 1

Crocce M., Scoccimarro R., 2006, Phys. Rev. D, 73, 063520

Cuesta A. J., Niro V., Verde L., 2016a, Phys. Dark Univ., 13, 77

Cuesta A. J. et al., 2016b, MNRAS, 457, 1770

Dawson K. S. et al., 2013, AJ, 145, 10

de Putter R. et al., 2012, ApJ, 761, 12

Dolgov A. D., 2002, Phys. Rep., 370, 333

Drinkwater M. J. et al., 2010, MNRAS, 401, 1429

Dunkley J. et al., 2009, ApJS, 180, 306

Eisenstein D. J., Hu W., 1998, ApJ, 496, 605

Eisenstein D. J. et al., 2005, ApJ, 633, 560

Eisenstein D. J., Seo H.-J., White M. J., 2007, ApJ, 664, 660

Eisenstein D. J. et al., 2011, AJ, 142, 72

Erben T. et al., 2013, MNRAS, 433, 2545

Feldman H. A., Kaiser N., Peacock J. A., 1994, ApJ, 426, 23

Fukugita M., Ichikawa T., Gunn J. E., Doi M., Shimasaku K., Schneider D. P., 1996, AJ, 111, 1748

Gil-Marín H. et al., 2016a, MNRAS, 460, 4188

Gil-Marín H. et al., 2016b, MNRAS, 460, 4210

Giusarma E., de Putter R., Ho S., Mena O., 2013, Phys. Rev. D, 88, 063515

Gong Y., Zhang T.-J., Lan T., Chen X.-L., 2008, preprint (arXiv:0810.3572)

Green J. et al., 2012, preprint (arXiv:1208.4012)

Grieb J. N. et al., 2017, MNRAS, 467, 2085

Gunn J. E. et al., 1998, AJ, 116, 3040

Gunn J. E. et al., 2006, AJ, 131, 2332

Hartlap J., Simon P., Schneider P., 2007, A\&A, 464, 399

Heymans C. et al., 2012, MNRAS, 427, 146

Hinshaw G. et al., 2009, ApJS, 180, 225

Hu W., Eisenstein D. J., Tegmark M., 1998, Phys. Rev. Lett., 80, 5255

Ichiki K., Takada M., Takahashi T., 2009, Phys. Rev. D, 79, 023520

Kaiser N., 1987, MNRAS, 227, 1

Kitaura F.-S., Hess S., 2013, MNRAS, 435, 78

Kitaura F.-S., Yepes G., Prada F., 2014, MNRAS, 439, 21

Kitaura F.-S., Gil-Marín H., Scoccola C., Chuang C.-H., Müller V., Yepes

G., Prada F., 2015, MNRAS, 450, 1836

Kitaura F.-S. et al., 2016a, Phys. Rev. Lett., 116, 171301

Kitaura F.-S. et al., 2016b, MNRAS, 456, 4156

Komatsu E. et al., 2011, ApJS, 192, 18

Landy S. D., Szalay A. S., 1993, ApJ, 412, 64

Laureijs R. et al., 2011, preprint (arXiv:1110.3193)

Leahy J. P. et al., 2010, A\&A, 520, A8

Lesgourgues J., Pastor S., 2006, Phys. Rep., 429, 307 
Lesgourgues J., Perotto L., Pastor S., Piat M., 2006, Phys. Rev. D, 73, 045021

Lewis A., 2013, Phys. Rev. D, 87, 103529

Lewis A., Bridle S., 2002, Phys. Rev. D, 66, 103511

Lewis A., Challinor A., 2002, Phys. Rev. D, 66, 023531

Lewis A., Challinor A., Lasenby A., 2000, ApJ, 538, 473

Li H., Liu J., Xia J.-Q., Sun L., Fan Z. H., Tao C., Tilquin A., Zhang X., 2009, Phys. Lett. B, 675, 164

Liang Y., Zhao C., Chuang C.-H., Kitaura F.-S., Tao C., 2016, MNRAS, 459,4020

Ma C.-P., Bertschinger E., 1995, ApJ, 455, 7

Matsubara T., 2008, Phys. Rev. D, 77, 063530

Mennella A. et al., 2011, A\&A, 536, A3

Mohayaee R., Mathis H., Colombi S., Silk J., 2006, MNRAS, 365, 939

Neyrinck M. C., 2013, MNRAS, 428, 141

Olive K., Group P. D., 2014, Chin. Phys. C, 38, 090001

Parkinson D. et al., 2012, Phys. Rev. D, 86, 103518

Perlmutter S. et al., 1999, ApJ, 517, 565

Planck Collaboration I, 2011, A\&A, 536, A1

Planck Collaboration I, 2016a, A\&A, 594, A1

Planck Collaboration XI, 2016b, A\&A, 594, A11

Planck Collaboration XIII, 2016c, A\&A, 594, A13

Planck Collaboration XV, 2016d, A\&A, 594, A15

Planck HFI Core Team, 2011, A\&A, 536, A4

Reid B. A., White M., 2011, MNRAS, 417, 1913

Reid B. A., Verde L., Jimenez R., Mena O., 2010, J. Cosmol. Astropart. Phys., 1001, 003

Reid B. A. et al., 2012, MNRAS, 426, 2719

Reid B. A., Seo H.-J., Leauthaud A., Tinker J. L., White M., 2014, MNRAS, 444, 476

Reid B. et al., 2016, MNRAS, 455, 1553

Riemer-Sørensen S., Parkinson D., Davis T. M., 2014, Phys. Rev. D, 89, 103505

Riess A. G. et al., 1998, AJ, 116, 1009

Rodríguez-Torres S. A. et al., 2016, MNRAS, 460, 1173

Ross A. J. et al., 2012, MNRAS, 424, 564

Ross A. J. et al., 2014, MNRAS, 437, 1109

Ross A. J. et al., 2017, MNRAS, 464, 1168

Rosset C. et al., 2010, A\&A, 520, A13

Rozo E., Rykoff E. S., Bartlett J. G., Evrard A. E., 2013, preprint (arXiv: 1302.5086)

Saito S., Takada M., Taruya A., 2011, Phys. Rev. D, 83, 043529

Sako M. et al., 2014, ApJS, preprint (arXiv:1401.3317)

Salazar-Albornoz S. et al., 2016, MNRAS, preprint (arXiv:1607.03144)

Samushia L. et al., 2014, MNRAS, 439, 3504

Sanchez A. G. et al., 2012, MNRAS, 425, 415

Sanchez A. G. et al., 2014, MNRAS, 440, 2692

Sanchez A. G. et al., 2017a, MNRAS, 464, 1493

Sanchez A. G. et al., 2017b, MNRAS, 464, 1640

Satpathy S. et al., 2016, MNRAS, preprint (arXiv:1607.03148)

Schlegel D. et al., 2011, preprint (arXiv:1106.1706)

Seljak U., Slosar A., McDonald P., 2006, J. Cosmol. Astropart. Phys., 0610, 014

Slepian Z., Eisenstein D. J., 2016, MNRAS, preprint (arXiv:1607.03109)

Slepian Z. et al., 2016, MNRAS, preprint (arXiv:1607.06097)

Slepian Z. et al., 2017, MNRAS, 468, 1070

Smee S. et al., 2013, AJ, 146, 32

Tauber J. A. et al., 2010, A\&A, 520, A1

Tereno I., Schimd C., Uzan J.-P., Kilbinger M., Vincent F. H., Fu L., 2009, A\&A, 500, 657

Thomas S. A., Abdalla F. B., Lahav O., 2010, Phys. Rev. Lett., 105, 031301

Tojeiro R. et al., 2014, MNRAS, 440, 2222

Van Waerbeke L., Mellier Y., 2003, preprint (astro-ph/0305089)

Vargas-Magaña M. et al., 2016, preprint (arXiv:1610.03506)

Wang Y., 2009, Phys. Rev. D, 80, 123525

Wang Y., 2014, MNRAS, 443, 2950

Wang Y., Mukherjee P., 2007, Phys. Rev. D, 76, 103533

Wang Y. et al., 2016, MNRAS, preprint (arXiv:1607.03154)
Wyman M., Rudd D. H., Vanderveld R. A., Hu W., 2014, Phys. Rev. Lett., 112,051302

Xia J.-Q. et al., 2012, J. Cosmol. Astropart. Phys., 1206, 010

York D. G. et al., 2000, AJ, 120, 1579

Zhao G.-B. et al., 2013, MNRAS, 436, 2038

Zhao G.-B. et al., 2017, MNRAS, 466, 762

${ }^{1}$ Instituto de Astrofísica de Canarias (IAC), C/Vía Láctea, s/n, E-38200 La Laguna, Tenerife, Spain

${ }^{2}$ Departamento Astrofísica, Universidad de La Laguna (ULL), E-38206 La Laguna, Tenerife, Spain

${ }^{3}$ Instituto de Física Teórica, (UAM/CSIC), Universidad Autónoma de Madrid, Cantoblanco, E-28049 Madrid, Spain

${ }^{4}$ Leibniz-Institut für Astrophysik Potsdam (AIP), An der Sternwarte 16, D-14482 Potsdam, Germany

${ }^{5}$ Institut de Ciències del Cosmos (ICCUB), Universitat de Barcelona (IEECUB), Martí i Franquès 1, E-08028 Barcelona, Spain

${ }^{6}$ National Astronomical Observatories, Chinese Academy of Sciences, Beijing 100012, China

${ }^{7}$ Institute of Cosmology and Gravitation, University of Portsmouth, Dennis Sciama Building, Portsmouth PO1 3FX, UK

${ }^{8}$ Center for Cosmology and Astroparticle Physics, Department of Physics, The Ohio State University, OH 43210, USA

${ }^{9}$ Campus of International Excellence UAM+CSIC, Cantoblanco, E-28049 Madrid, Spain

${ }^{10}$ Departamento de Física Teórica M8, Universidad Autonoma de Madrid (UAM), Cantoblanco, E-28049 Madrid, Spain

${ }^{11}$ Instituto de Astrofísica de Andalucía (CSIC), Glorieta de la Astronomía, E-18080 Granada, Spain

${ }^{12}$ Brookhaven National Laboratory, Upton, NY 11973, USA

${ }^{13}$ Department of Physics, Carnegie Mellon University, 5000 Forbes Ave., Pittsburgh, PA 15213, USA

${ }^{14}$ The McWilliams Center for Cosmology, Carnegie Mellon University, 5000 Forbes Ave., Pittsburgh, PA 15213, USA

${ }^{15}$ Lawrence Berkeley National Lab, 1 Cyclotron Rd, Berkeley, CA 94720 , USA

${ }^{16}$ Harvard-Smithsonian Centerfor Astrophysics, 60 Garden St., Cambridge, MA 02138, USA

${ }^{17}$ Sorbonne Universités, Institut Lagrange de Paris (ILP), 98 bis Boulevard Arago, F-75014 Paris, France

${ }^{18}$ Laboratoire de Physique Nucléaire et de Hautes Energies, Université Pierre et Marie Curie, 4 Place Jussieu, F-75005 Paris, France

${ }^{19}$ Universitäts-Sternwarte München, Scheinerstrasse 1, D-81679 Munich, Germany

${ }^{20}$ Max-Planck-Institut für extraterrestrische Physik, Postfach 1312, Giessenbachstr., D-85741 Garching, Germany

${ }^{21}$ Departments of Physics and Astronomy, University of California, Berkeley, CA 94720, USA

${ }^{22}$ Department of Physics and Astronomy, Sejong University, Seoul 143-747, Korea

${ }^{23}$ Kansas State University, Manhattan, KS 66506, USA

${ }^{24}$ National Abastumani Astrophysical Observatory, Ilia State University, $2 \mathrm{~A}$ Kazbegi Ave., GE-1060 Tbilisi, Georgia

${ }^{25}$ Department of Physics and Astronomy, Ohio University, 251B Clippinger Labs, Athens, OH 45701, USA

${ }^{26}$ Center for Cosmology and Particle Physics, Department of Physics, New York University, 4 Washington Place, New York, NY 10003, USA

${ }^{27}$ School of Physics and Astronomy, University of St Andrews, St Andrews, KY16 9SS, UK

${ }^{28}$ Instituto de Fisica, Universidad Nacional Autónoma de México, Ciudad de México, Apdo. Postal 20-364, México

${ }^{29}$ Department of Physics and Astronomy, University of Utah, 115 S 1400 E, Salt Lake City, UT 84112, USA

${ }^{30}$ Department of Chemistry and Physics, King's College, 133 North River St, Wilkes Barre, PA 18711, USA

This paper has been typeset from a $\mathrm{T}_{\mathrm{E}} \mathrm{X} / \mathrm{LAT} \mathrm{E} \mathrm{X}$ file prepared by the author. 